Revue des patrimoines

34 | 2018

Lieux de pouvoirs. Architectures administratives dans la France contemporaine, 1945-2013. Avant/Après la décentralisation

\title{
La préfecture du Val-d'Oise édifiée par Henry Bernard à Cergy-Pontoise
}

Un geste architectural «parachuté en pleins champs » ou la tentative d'« affirmation d'un nouveau type d'intervention de l'administration auprès des citoyens "

The Val-d'Oise Prefecture building, designed by Henry Bernard at Cergy-

Pontoise

Léo Noyer-Duplaix et Emmanuelle Philippe

\section{OpenEdition}

Édition électronique

URL : http://journals.openedition.org/insitu/16024

DOI : $10.4000 /$ insitu. 16024

ISSN : 1630-7305

Éditeur

Ministère de la culture

Référence électronique

Léo Noyer-Duplaix et Emmanuelle Philippe, «La préfecture du Val-d'Oise édifiée par Henry Bernard à Cergy-Pontoise », In Situ [En ligne], 34 | 2018, mis en ligne le 23 mai 2018, consulté le 03 mai 2019. URL : http://journals.openedition.org/insitu/16024 ; DOI : 10.4000/insitu.16024

Ce document a été généré automatiquement le 3 mai 2019.

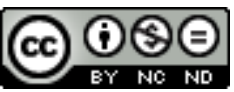

In Situ Revues des patrimoines est mis à disposition selon les termes de la licence Creative Commons Attribution - Pas d'Utilisation Commerciale - Pas de Modification 4.0 International. 


\section{La préfecture du Val-d'Oise édifiée par Henry Bernard à Cergy-Pontoise}

Un geste architectural « parachuté en pleins champs » ou la tentative d'« affirmation d'un nouveau type d'intervention de l'administration auprès des citoyens »

The Val-d'Oise Prefecture building, designed by Henry Bernard at Cergy-

Pontoise

Léo Noyer-Duplaix et Emmanuelle Philippe

La réorganisation administrative de la région parisienne, décidée au milieu des années 1960, conduisit à la création du département du Val-d'Oise et à l'édification d'une nouvelle préfecture (fig. 1) dont les plans furent confiés à l'architecte Henry Bernard assisté de Pierre Mougin et de Robert Décosse. L'édifice constitua, comme le rappela Bernard, «le premier geste de l'État », « parachuté en pleins champs avant toute autre réalisation » et qui se "devait donc d'être à tout point de vue symbolique : symbolique par sa situation, par son esprit, par sa structure, et par les techniques de pointe utilisées » avec " pour objectif l'affirmation d'un nouveau type d'intervention de l'administration auprès des citoyens ${ }^{1}$. Élément majeur de la ville nouvelle de Cergy-Pontoise, la préfecture, qui fut érigée sur la commune de Cergy au sud du quartier éponyme urbanisé sur dalle, domine un parc urbain de huit hectares aménagé par Alain Provost. L'édifice témoigne aujourd'hui de la volonté de renouvellement d'une typologie. 
Figure 1

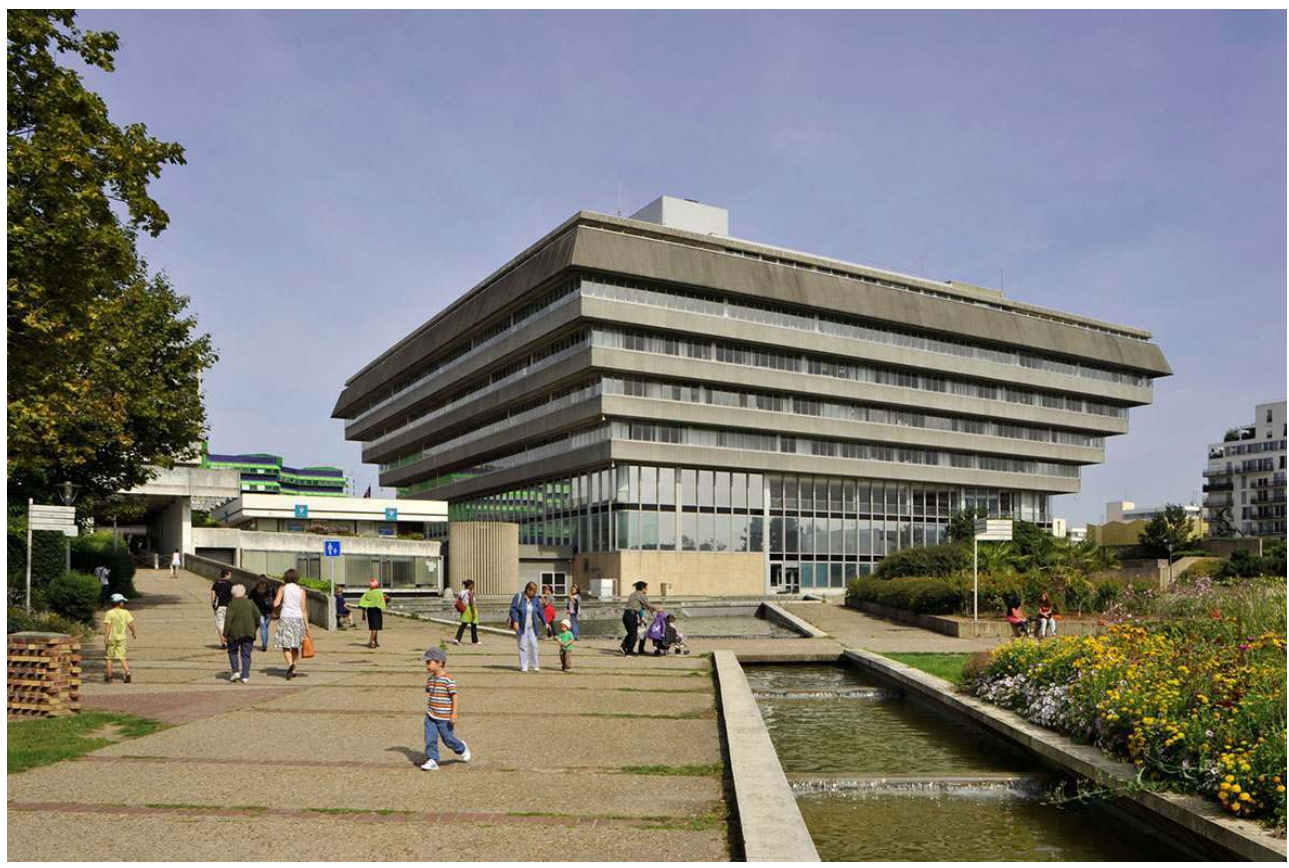

La préfecture depuis le parc

Phot. Laurent Kruszyk. @ Région île-de-France/Laurent Kruszyk/ADAGP 2015.

2 Il s'agit donc de comprendre, outre les circonstances qui présidèrent la conception de la préfecture, quel fut son parti constructif et architectural pour enfin appréhender les principes d'une volonté de dépassement du cadre administratif.

Cette monographie de la préfecture du Val-d'Oise constitue l'une des restitutions de l'étude du quartier urbanisé sur dalle de Cergy-Préfecture conduite par le service Patrimoines et Inventaire de la Région Île-de-France. Elle s'inscrit également dans le cadre d'une thèse de doctorat menée à Sorbonne Université et portant sur la carrière d'architecte et d'urbaniste d'Henry Bernard 2 . Elle est fondée sur le dépouillement de la presse spécialisée, des archives de l'architecte - conservées au Centre d'archives d'architecture du $\mathrm{xx}^{\mathrm{e}}$ siècle - et du fonds Bernard Hirsch - conservé aux archives départementales du Val-d'Oise. Déposés auprès de ces dernières mais non communicables lors de nos recherches, les documents concernant la construction de la préfecture (966 W 1-69), fonds de la direction départementale de l'Équipement, n'ont pu être consultés.

\section{«Une préfecture aux champs »}

\section{Aux origines de la ville nouvelle}

La loi du 10 juillet 1964 portant réorganisation de la région parisienne vint bouleverser le territoire francilien en le restructurant administrativement, et ce, dans un dessein tant géographique et urbanistique que politique ${ }^{3}$. Les départements de la Seine, de la Seine-etOise et de la Seine-et-Marne furent ainsi dissous afin de donner officiellement naissance le $1^{\text {er }}$ janvier 1968 aux départements des Hauts-de-Seine, de la Seine-Saint-Denis, du Valde-Marne, de l'Essonne, des Yvelines et du Val-d'Oise. Le haut fonctionnaire Paul 
Delouvrier (1914-1995) fut, en tant que délégué général au District de Paris de 1961 à 1969, chargé par le pouvoir gaulliste d'orchestrer cette réorganisation. En juin 1965, il rendit public le Schéma directeur d'aménagement et d'urbanisme de la région de Paris (SDAURP), dont la création de villes nouvelles constituait l'élément majeur.

5 Le Val-d'Oise, nouvelle entité administrative issue de la partie nord de l'ancien département de la Seine-et-Oise, se cherchait alors un chef-lieu. Le château de Marcouville à Pontoise ou l'abbaye de Maubuisson à Saint-Ouen-l'Aumône furent envisagés. Mais Paul Delouvrier en décida autrement et désigna, lors d'un survol de la boucle de l'Oise en hélicoptère ${ }^{4}$, les champs de betteraves situés à mi-chemin entre la ville de Pontoise et le village de Cergy pour accueillir le quartier de la Préfecture ${ }^{5}$. Centre fédérateur de « Pontoise-Cergy »- l'appellation « Cergy-Pontoise » ne fut employée qu'à partir de 1969 - ville nouvelle destinée à accueillir 300000 habitants, ce quartier créé ex nihilo fut défini par le SDAURP comme le pôle du nord-ouest de la région parisienne (fig. 2). Cœur du département nouveau et de la ville nouvelle, il devait constituer un premier geste fort d'urbanisme de l'État qui y édifia, au milieu des champs, la préfecture. Il est à noter toutefois que bien qu'abritant aujourd'hui les bureaux de l'administration préfectorale du Val-d'Oise, le quartier de la préfecture, situé sur la commune de Cergy, n'est pas le chef-lieu départemental. Celui-ci fut en effet fixé par le décret du 16 avril 1969 à Pontoise qui demeure depuis le chef-lieu du Val-d'Oise alors que la ville n'est qu'une sous-préfecture. Cette situation est un cas unique en France.

Figure 2

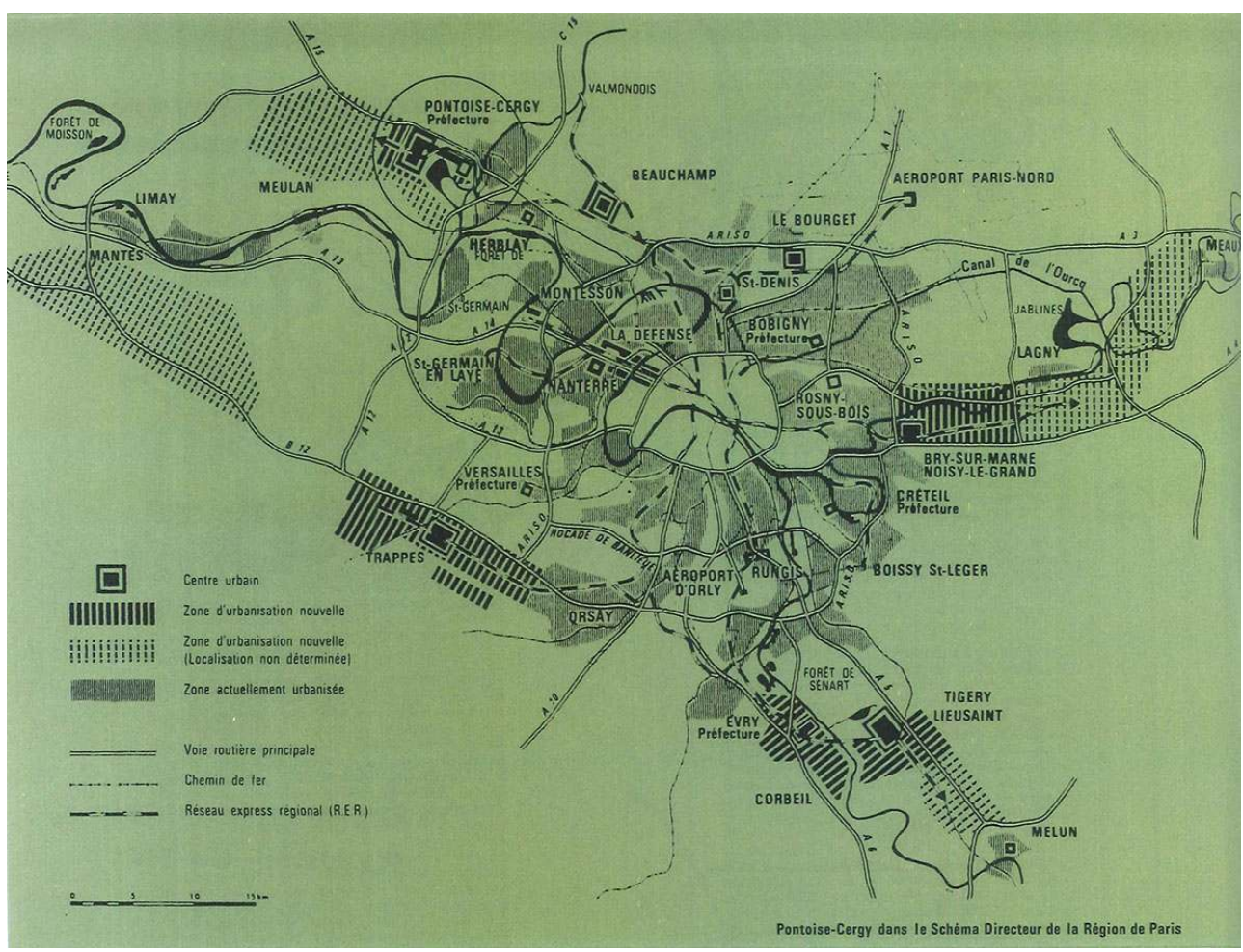

La ville nouvelle de Pontoise-Cergy dans le Schéma directeur d'aménagement et d'urbanisme de la région de Paris (extrait de Urbanisme, n¹05, 1968, p. 34).

Repro. Léo Noyer-Duplaix. (c) Droits réservés.

6 Afin de mener à bien le chantier de la ville nouvelle, Paul Delouvrier chargea dès l'été 1965 l'ingénieur des Ponts et Chaussées Bernard Hirsch (1927-1988) ${ }^{6}$ de travailler sur 
l'urbanisme cergypontain. Figure majeure du projet, l'ingénieur s'entoura de l'urbaniste et architecte Jean Coignet (1928-2001) ${ }^{7}$ - qui fut à l'origine, selon Hirsch, du principe et de la silhouette de la ville nouvelle - et de l'architecte Michel Folliasson (1925-2011) ${ }^{8}$. Enfin, André Chadeau (1927-2017) fut nommé le 19 septembre 1964 préfet délégué du Val-d'Oise, poste qu'il occupa jusqu'au 13 septembre 1967. L'intervention de Chadeau dans la conception de la nouvelle préfecture fut décisive - la volonté, notamment, de renouveler les principes de l'architecture administrative - et coïncida d'ailleurs avec la phase d'élaboration de l'édifice.

$7 \quad$ L'édification de la préfecture étant le premier chantier de la ville nouvelle (fig. 3), le travail de la Mission d'aménagement dirigée par Bernard Hirsch fut singulier. Il ne s'agissait pas en effet d'un raisonnement qui procédait de l'ensemble au particulier, du plan général de la ville au premier quartier dans lequel le siège de l'administration préfectorale s'intégrerait. Devant «travailler simultanément à toutes les échelles ${ }^{9}$ ", la Mission se concentra d'abord sur l'étude précise des abords de la préfecture dont la conception fut confiée à l'architecte Henry Bernard.

Figure 3

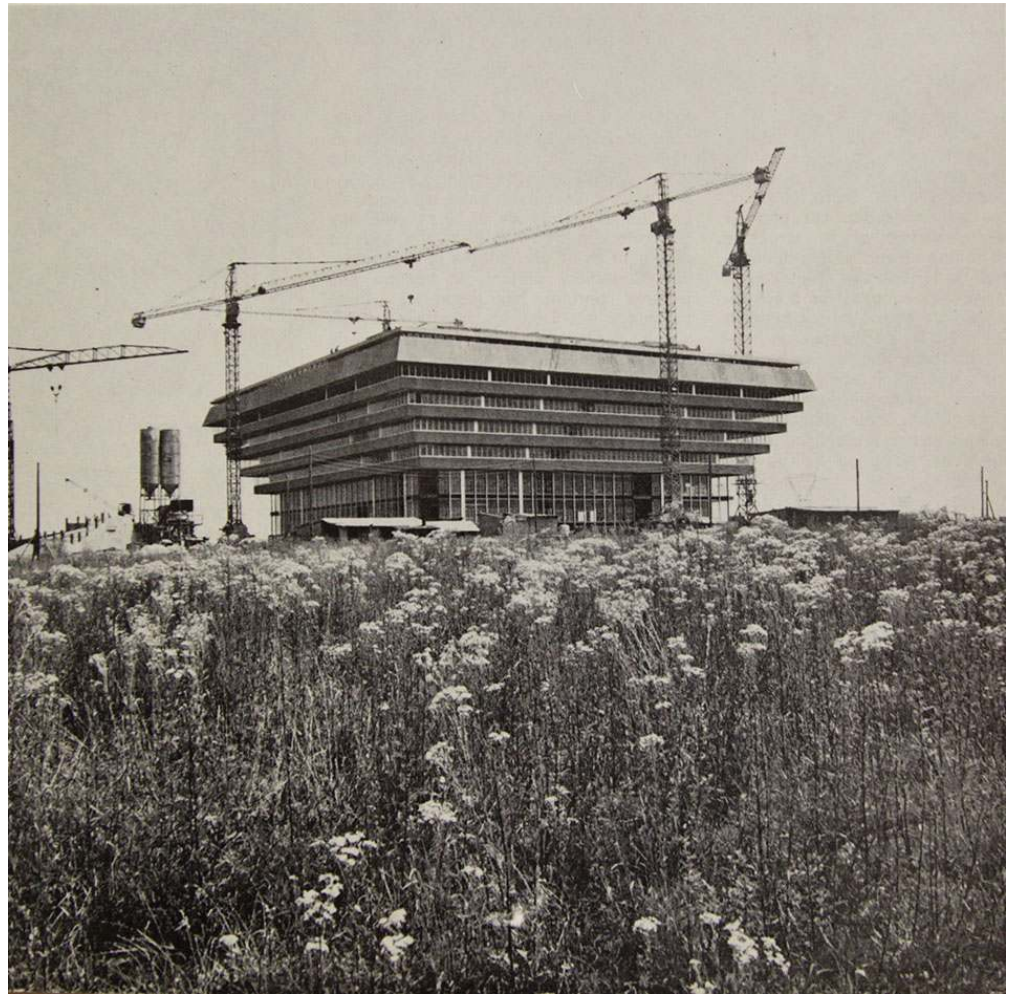

Le chantier de la préfecture au milieu des champs, 1969 (archives de l'IFA ; fonds Bernard 266 AA).

Repro. Léo Noyer-Duplaix. ( ) Académie d'architecture/Cité de l'architecture et du patrimoine/Archives d'architecture du XXe siècle/ADAGP.

\section{Henry Bernard et la « Préfecture 1970 »}

Le gouvernement gaulliste souhaitait marquer la construction des préfectures dans les départements nouveaux de la région parisienne d'un grand geste qui affirmerait l'autorité de l'État dans ces territoires. Ce fut André Malraux, alors ministre d'État chargé 
des Affaires culturelles, qui, sur la proposition de Paul Delouvrier, choisit les architectes des nouveaux édifices. À Cergy, le ministre désigna Henry Bernard (fig. 4).

Figure 4

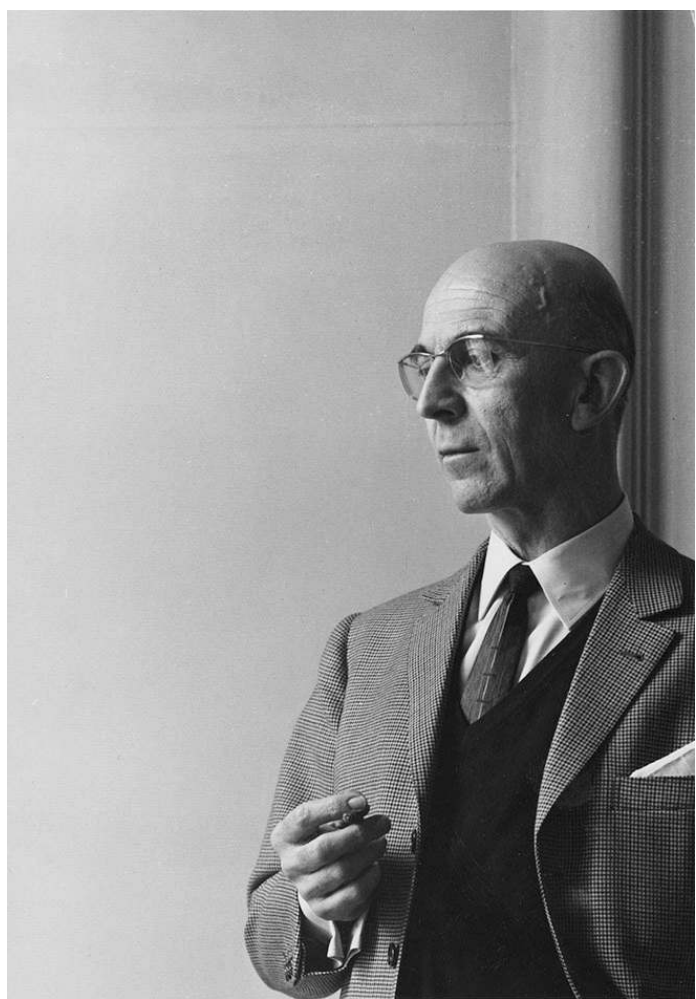

Portrait d'Henry Bernard (archives de l'IFA ; fonds Bernard 266 AA)

Repro. Archives d'architecture du Xxe siècle. (c) Académie d'architecture/Cité de l'architecture et du patrimoine/Archives d'architecture du Xxe siècle/ADAGP.

Né le 21 février 1912 à Albertville (Savoie) et mort le 10 décembre 1994 à Paris, fils d'un polytechnicien, Henry Bernard intégra l'École des beaux-arts et l'atelier de Paul Bigot (1870-1942 $)^{10}$ en juin 1930. Lauréat en 1938 du premier grand prix de Rome, l'architecte eut son séjour à la Villa Médicis écourté par sa mobilisation sur la frontière belge où il fut fait prisonnier en mai 1940. Retenu à Stablack (Prusse orientale), il consacra ses années de captivité à l'étude de l'urbanisme parisien. À la Libération, il œuvra à la reconstruction de la ville de Caen, à travers l'édification de l'université, en particulier - unique université française de la reconstruction, classée au titre des Monuments historiques le 15 mai 2012 ${ }^{11}$ - et de l'église paroissiale Saint-Julien - classée le 27 juin $2007^{12}$. En 1953, sa carrière prit un tournant lorsqu'il fut lauréat du concours lancé par la Radiodiffusion-télévision française (RTF) pour la construction de la Maison de la Radio à Paris, témoignage majeur de l'architecture française des Trente Glorieuses. Inauguré le 14 décembre 1963 par le général de Gaulle, ce colossal bâtiment constituait un symbole de la puissance étatique. L'architecture hospitalière tint en outre une importante place dans sa carrière. Il conçut notamment, suite à la réforme Debré de 1958 qui instituait les centres hospitaliers et universitaires (CHU), un parti de construction hospitalo-universitaire type qu'il mit en œuvre à Caen ${ }^{13}$. Enfin, de 1972 à 1977, Henry Bernard se consacra à la construction d'une nouvelle incarnation de l'autorité publique, le palais de l'Europe à Strasbourg. 
10 Arrivé dans la profession par la "voie royale ", c'est-à-dire le premier grand prix de Rome, Bernard accéda aux plus hautes fonctions, reçut de nombreux titres, et le 23 octobre 1968, fut élu membre de l'Académie des beaux-arts. Comme le rappelle l'historien François Loyer, Henry Bernard appartenait « à l'élite restreinte des architectes en charge de la commande publique », leur pouvoir «ayant été sans partage jusqu'à la réforme de 1968 ». Loyer indique également avec justesse que « rien ne manifeste plus clairement l'assimilation du langage moderniste par l'École des beaux-arts que la carrière qui lui a été réservée jusqu'à la fin des années soixante $»^{14}$.

De sa formation aux Beaux-Arts, Henry Bernard garda une sensibilité classique, un goût pour la composition ainsi qu'un questionnement constant sur la monumentalité qui traversa toute son œuvre, tant urbanistique qu'architecturale. Cette persistante redéfinition du monument - qui se manifesta notamment par un travail de recherche sur les formes architecturales - rejoignait des convictions politiques royalistes affichées l'architecte écrivit à de nombreuses reprises dans l'hebdomadaire royaliste La Nation française $^{15}$. Pour Bernard, les édifices de la Nation - il n'employait jamais le terme de République - devaient conserver un caractère sacré. Il en fut ainsi, comme le souligna Bernard Hirsch, lors de la conception de la préfecture du Val-d'Oise :

Henry Bernard avait, sur la symbolique de la fonction préfectorale, des idées très arrêtées, qui s'accordaient d'ailleurs à son tempérament. L'autorité devait s'exercer dans la noblesse, dans le recueillement et dans la solennité. Plusieurs partis avaient été esquissés avant d'aboutir à la pyramide renversée. Ils avaient en commun le souci d'entourer les bâtiments administratifs par des douves infranchissables comme des enceintes médiévales ${ }^{16}$.

Mais cette conception de l'incarnation architecturale de la puissance publique n'était pas partagée par le préfet délégué André Chadeau - rejoint dans ses propos par Jean Coignet, qui orienta les premières études d'urbanisme dans son sens - qui souhaitait, comme le rappela Bernard Hirsch, «abattre les barrières entre l'Administration et les usagers et faire de la préfecture un lieu d'animation et de rencontre où il serait possible à l'occasion d'une formalité administrative d'acheter son journal, de boire un pastis ou d'écouter de la musique $\aleph^{17}$. Du reste, grâce à la lettre de mission d'André Malraux, Henry Bernard disposait d'une grande indépendance, obligeant les membres de la Mission d'aménagement à faire preuve « de beaucoup de diplomatie pour donner à la préfecture l'image de la nouvelle administration à laquelle André Chadeau tenait beaucoup ${ }^{18}$. La conception puis la réalisation de la préfecture du Val-d'Oise furent ainsi le lieu d'un constant dialogue - que Bernard qualifia de «fertile $»^{19}$ bien qu'il fût parfois difficile entre l'architecte et le préfet délégué. Malgré leurs divergences de vues, les deux hommes cherchèrent à concevoir un édifice exemplaire. Bernard souhaita même que l'édifice cergypontain fasse date dans ce type de programme en devenant la "Préfecture 1970 » (fig. 5) :

(...) ce qui en effet m'intéresse au premier chef dans tous les projets que je suis amené à faire, c'est d'une part que le thème en soit différent (par exemple, une Université, une Maison de la Radio, une église, une Ambassade, une Préfecture, un plan d'Urbanisme) j'ai tenté pour chacun d'eux, non seulement de traiter le problème particulier localisé, mais aussi d'apporter une solution type à chacun de ces programmes. J'ai essayé de faire de la Préfecture de Pontoise, la «Préfecture $1970 »^{20}$. 
Figure 5

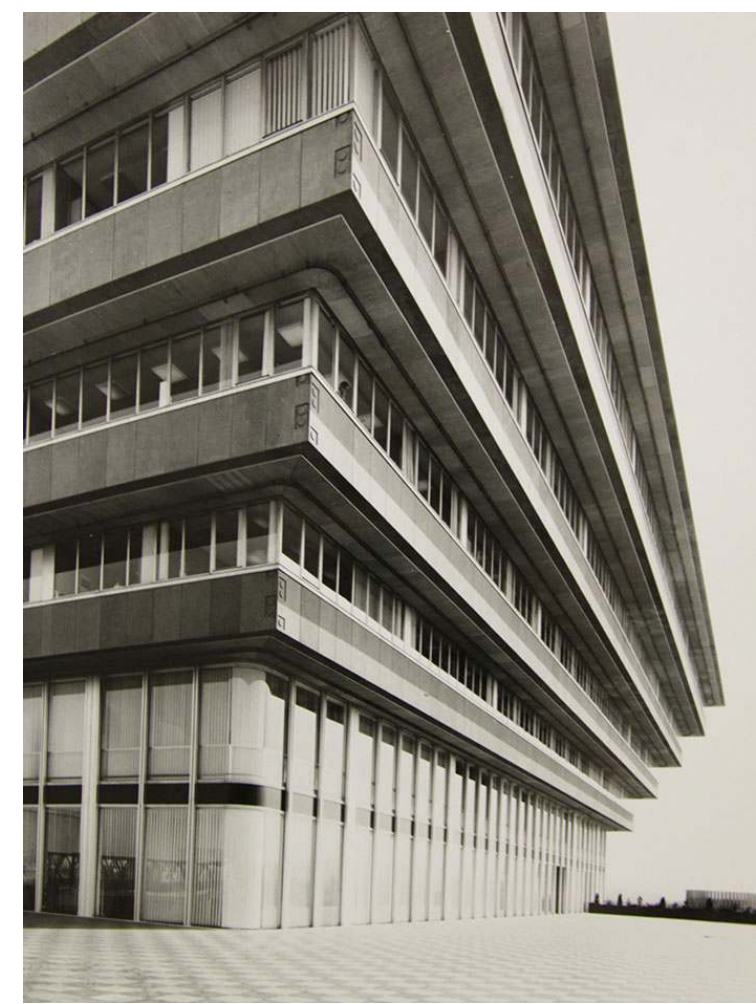

La préfecture peu après son édification, vers 1970 (archives de l'IFA ; fonds Bernard 266 AA).

Repro. Léo Noyer-Duplaix. (c) Académie d'architecture/Cité de l'architecture et du patrimoine/Archives d'architecture du xxe siècle/ADAGP.

Le programme de la nouvelle préfecture du Val-d'Oise fut élaboré à partir des besoins retenus en Comité interministériel et définitivement fixés par arbitrage du Premier ministre début $1965^{21}$. Les premières études conduites cette même année prévoyaient un édifice «carré bas, à façades verticales disposées autour d'un patio central, assez classique, et destiné à s'intégrer à un plan de masse orchestré par rapport à une dalle surélevée de circulation pour les piétons $»^{22}$. Mais ce projet fut finalement abandonné " au bénéfice d'une option simplifiée se limitant, pour des raisons d'ordre territorial, à une préfecture étoffée ainsi qu'à une tour de bureaux, et procédant par étapes en fonction des crédits disponibles $»^{23}$. L'architecte eut ainsi la charge de réaliser un ensemble «conçu pour la satisfaction des besoins administratifs de $1985 »^{24}$ et qui se composait de deux tranches, la préfecture et une cité administrative qui prenait la forme d'une tour de bureaux - finalement non réalisée (fig. 6). Des différentes études survit donc la préfecture à laquelle l'architecte donna la forme d'une pyramide inversée en fondant son parti sur l'encorbellement. 


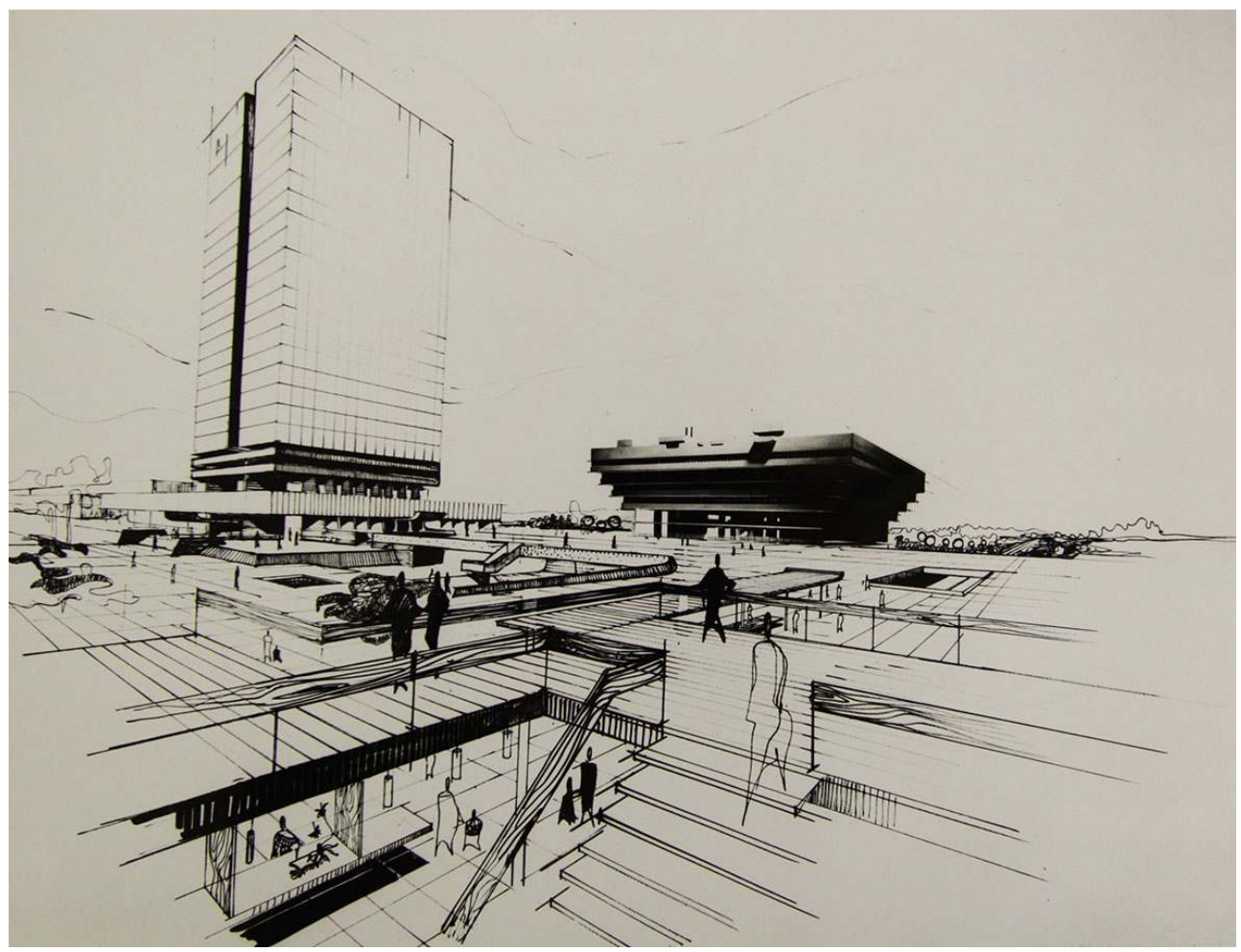

Les deux édifices initialement prévus, à droite la préfecture et à gauche la cité administrative, 1966 (archives de l'IFA; fonds Bernard 266 AA).

Repro. Léo Noyer-Duplaix. @ Académie d'architecture/Cité de l'architecture et du patrimoine/Archives d'architecture du XXe siècle/ADAGP.

\section{Une pyramide inversée}

\section{Le parti de l'encorbellement}

Le 29 mars 1967, des agriculteurs opposés à l'expropriation de leurs terres occupèrent le terrain destiné à l'édification de la préfecture. Le 14 juillet, ils furent évacués et la construction de ce vaste édifice débuta, pour s'achever le 2 juillet 1970, jour de son inauguration par Raymond Marcellin, alors ministre de l'Intérieur. Le chantier fut notamment marqué par l'édification - en raison de la forme en encorbellement - d'un vaste échafaudage de 100 kilomètres de tubes métalliques (fig. 7). Cet emmaillotement de métal ressemblait, selon Bernard Hirsch, à « une gravure de Bernard Buffet ${ }^{25}$ ». En outre, contrairement à ce qui est souvent avancé, la préfecture du Val-d'Oise ne fut pas le "premier immeuble construit de la ville nouvelle de Cergy-Pontoise ${ }^{26}$ ». Si son chantier fut en effet le premier à démarrer, le premier édifice livré fut le siège de la Mission d'aménagement, conçu par Henry Mathé et Jean Coignet et achevé en mai $1968^{27}$. 
Figure 7

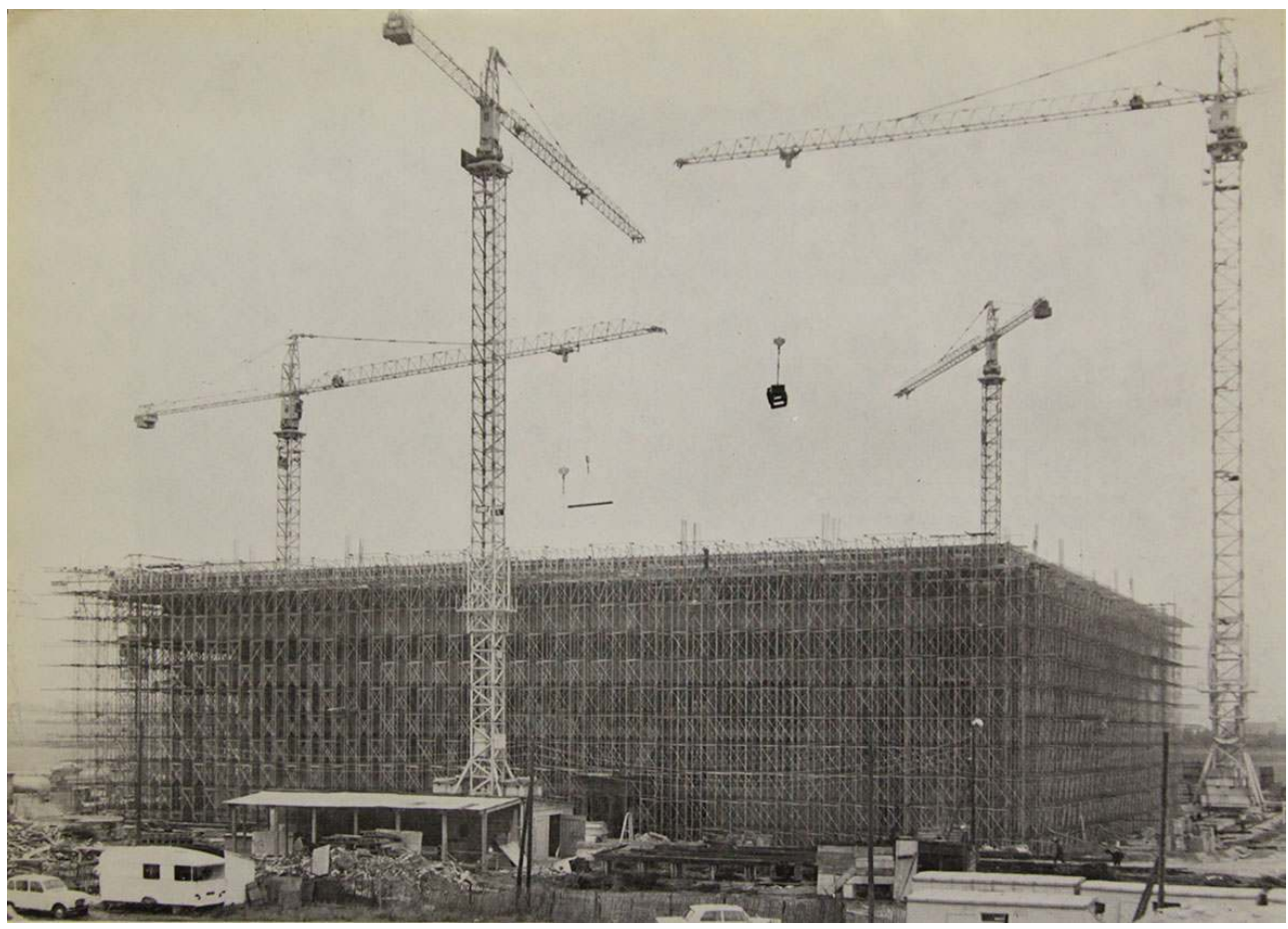

L'échafaudage de 100 km de tubes métalliques, vers 1968 (archives de l'IFA; fonds Bernard 266 AA).

Repro. Léo Noyer-Duplaix. (C) Académie d'architecture/Cité de l'architecture et du patrimoine/Archives d'architecture du XXe siècle/ADAGP.

La préfecture prend donc la forme d'une pyramide inversée édifiée sur une base carrée de 55,80 mètres pour un couronnement de 73,50 mètres. Au-dessus d'un socle, quatre étages sont construits en encorbellement sur le précédent, ce qui crée une succession de balcons qui s'ouvrent sur un vide central. Les quatre angles sont occupés par des tours qui abritent les circulations verticales de fluides et de personnes (fig. 8). D'une surface de 26700 mètres carrés hors œuvre, l'édifice s'élève à 28,33 mètres au niveau de terrasse des tours. 


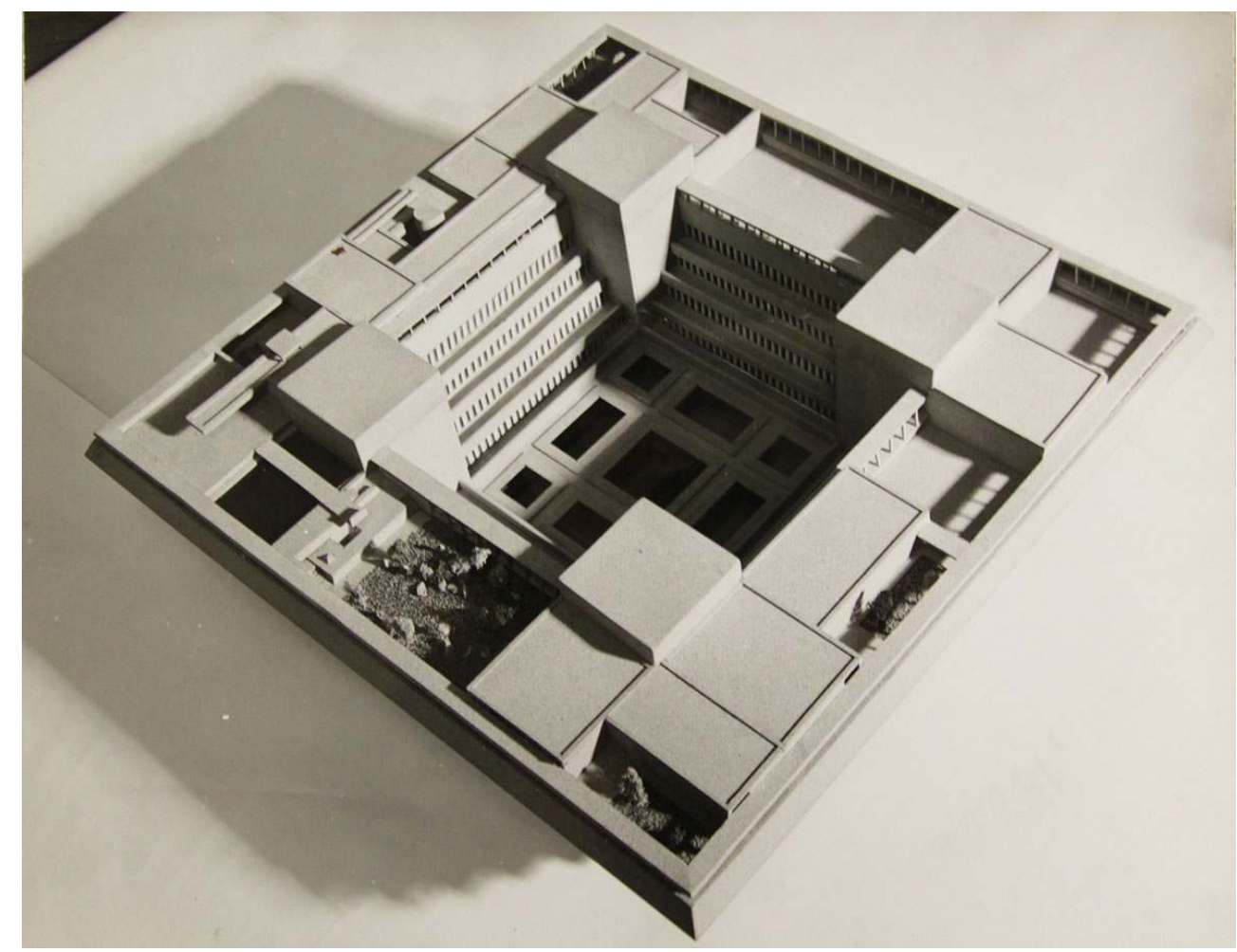

La maquette de la préfecture, vers 1967 (archives de l'IFA ; fonds Bernard 266 AA).

Repro. Léo Noyer-Duplaix. (c) Académie d'architecture/Cité de l'architecture et du patrimoine/Archives d'architecture du XXe siècle/ADAGP.

Le socle comporte un niveau en infrastructure, occupé par des espaces techniques, et trois niveaux en superstructure, les deux premiers constituant les rez-de-chaussée, c'està-dire le « rez-de-place » qui donne sur la dalle et le « rez-de-jardin » qui s'ouvre sur le $\operatorname{parc}^{28}$. Le public accède ainsi aujourd'hui à l'édifice par son entrée principale située au nord-est en «rez-de-place», et qui conduit au grand hall. Ce dernier, structurellement indépendant du reste du bâtiment, prend la forme d'un vaste atrium couvert par un "plancher-champignon » reposant sur quatre piles et constitué de caissons préfabriqués de béton qui en leur centre laissent place à des lanterneaux. Ce « plancher-terrasse » est aujourd'hui surmonté d'un système d'extraction de fumée de construction récente qui est surnommé, du fait de son esthétique, « le Beaubourg » (fig. 9). 
Figure 9

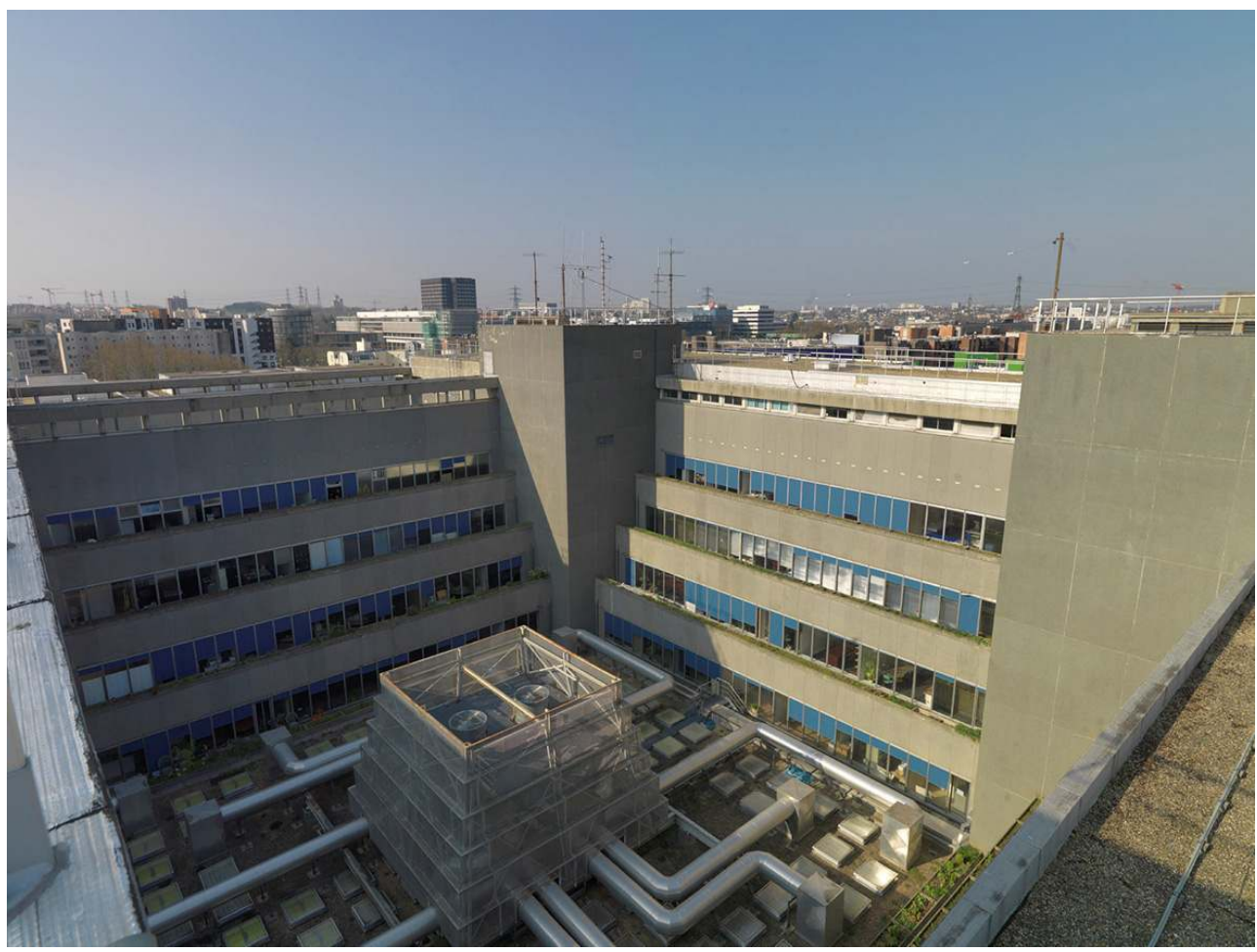

Le vide central de l'édifice.

Phot. Laurent Kruszyk. @ Région île-de-France/Laurent Kruszyk/ADAGP 2015.

17 Dans l'atrium, des escaliers relient le « rez-de-place » à la corbeille au « rez-de-jardin » qui s'ouvre largement sur le parc urbain. Le premier étage, qualifié par Bernard d'« étage noble ${ }^{29}$ ", abrite au nord-ouest le cabinet du préfet, au sud-est la salle des délibérations du conseil général, ces deux espaces étant reliés par des salons de réception qui sont en mezzanine sur le hall (fig. 10). 
Figure 10

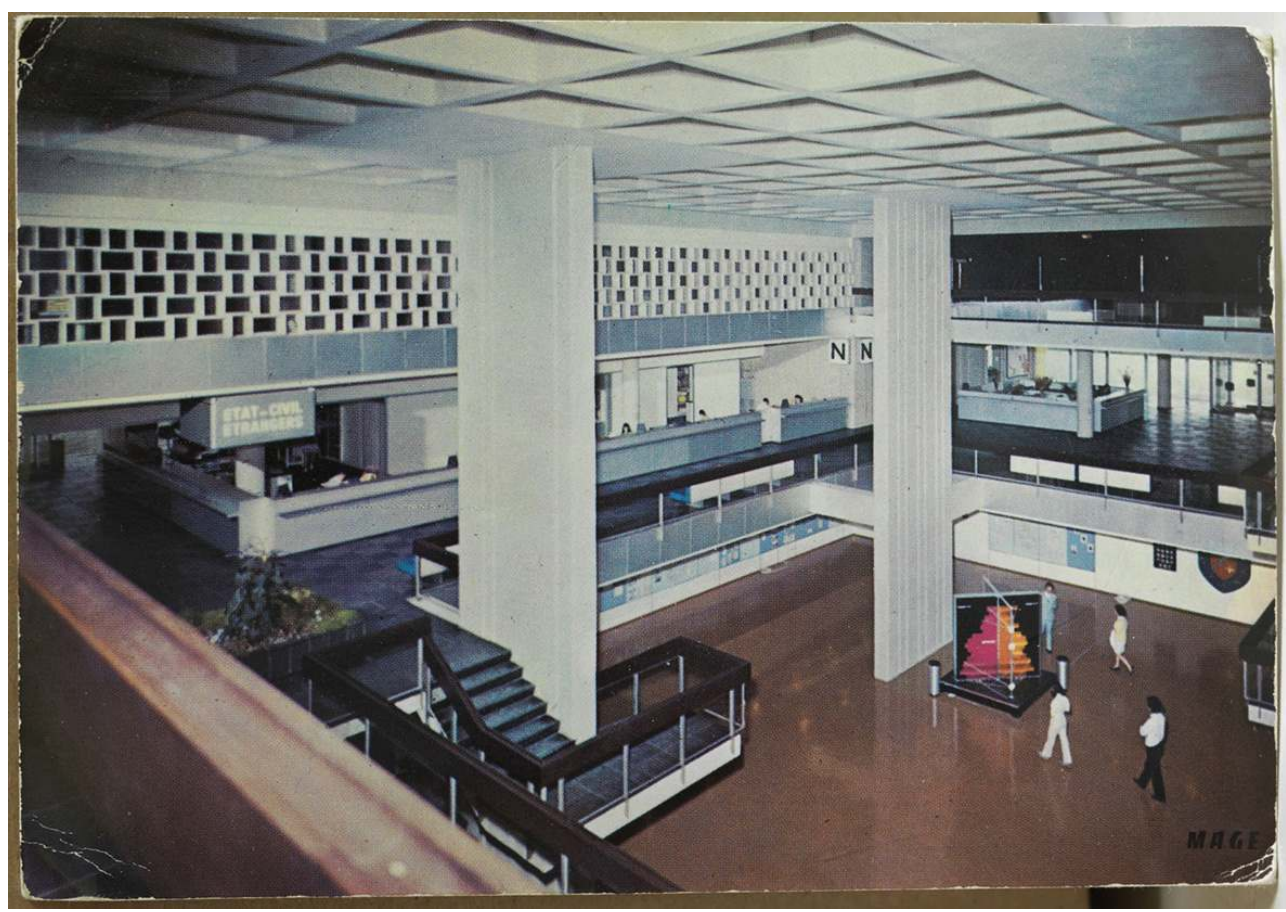

Le hall de la préfecture, carte postale, vers 1970 (archives de la préfecture du Val-d'Oise).

Repro. Laurent Kruszyk. @ Région Île-de-France/Laurent Kruszyk/ADAGP 2015.

Ainsi, pour l'architecte, ce socle, dans lequel prennent place les espaces réservés au public ainsi que le niveau «de commandement », résumait «le nouveau style d'intervention de l'autorité » :

(...) il est destiné à recevoir et à mettre au contact immédiat et permanent du public, non seulement des organes de renseignements divers (hôtesse, affichage, etc.), mais un échelon avancé de certains services et même des services complets permettant d'accomplir immédiatement certaines formalités administratives (carte grise, permis de conduire, passeport...) évitant ainsi des mouvements du public aux étages de bureaux ${ }^{30}$.

Le hall centralisant toutes les démarches, le public ne doit donc pas parcourir tout l'édifice afin d'effectuer telle ou telle formalités. Au-dessus du socle s'échelonnent quatre niveaux de bureaux, en porte-à-faux successifs de 1,80 mètre, suivant la trame de l'ossature. Ces bureaux à cloisons amovibles sont desservis par un "couloir-galerie » continu. Enfin, le niveau supérieur abrite, dans le couronnement d'une large corniche, trois appartements destinés au préfet, à son chef de cabinet et au secrétaire général ${ }^{31}$. L'ensemble de cet étage est aujourd'hui occupé par des bureaux, le préfet résidant à Pontoise, dans l'ancienne sous-préfecture ${ }^{32}$. Les appartements étaient ouverts sur des patios plantés, partageaient une piscine et jouissaient « d'une vue panoramique sur un horizon exceptionnel, notamment à l'ouest avec le futur plan d'eau et les hauteurs de l'Hautil $»^{33}$ (fig. 11). 


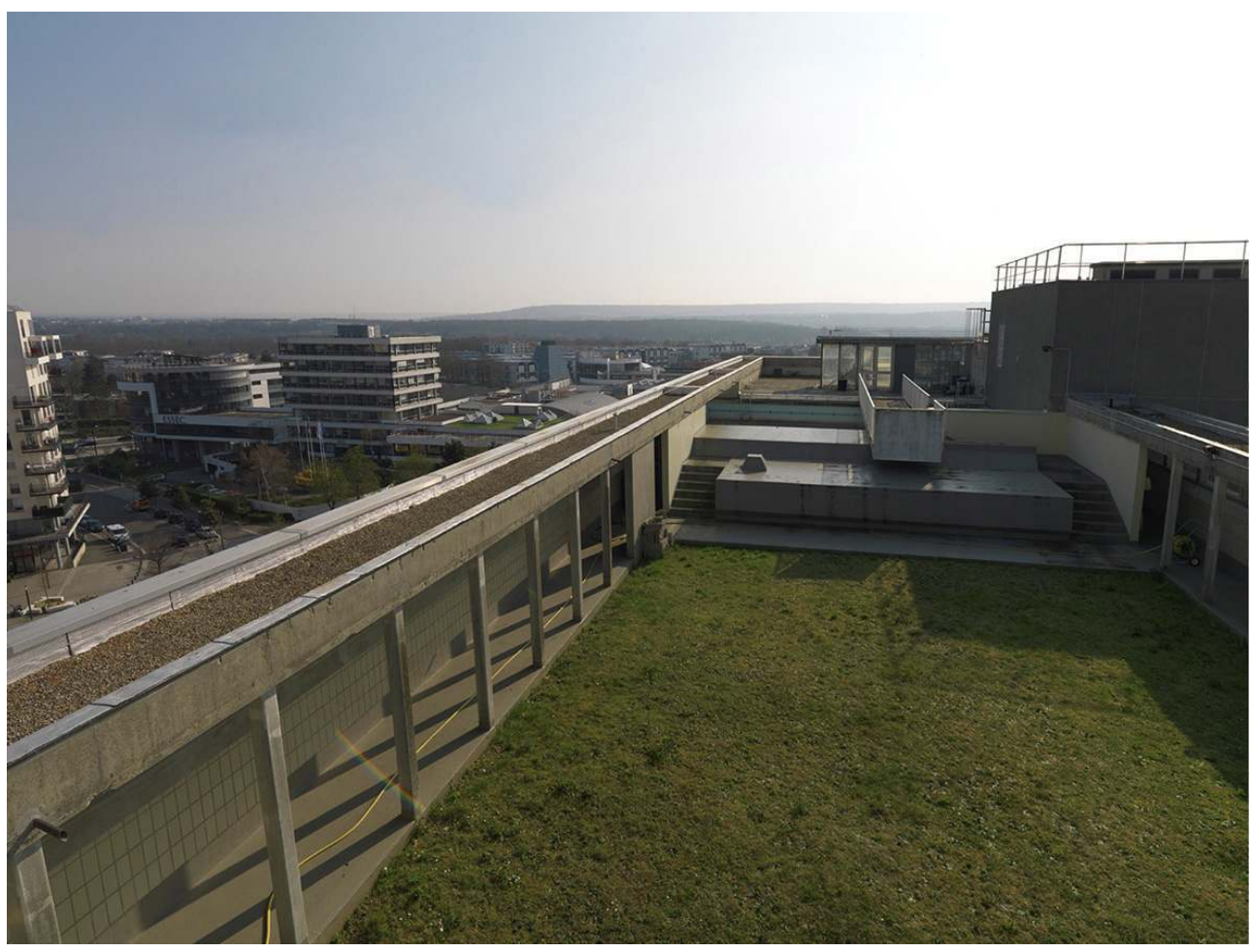

Au premier plan un des patios, au second la piscine aujourd'hui désaffectée, au loin les hauteurs de I'Hautil.

Phot. Laurent Kruszyk. @ Région Île-de-France/Laurent Kruszyk/ADAGP 2015.

20 Les huisseries extérieures, qui prennent la forme de baies en bandeaux, sont en aluminium anodisé. L'ensemble du bâtiment est en béton précontraint et, afin d'avoir une conception modulaire et de faciliter l'industrialisation de sa construction, la trame de son ossature est de 1,80 mètre. Henry Bernard l'explicita ainsi :

Rigoureusement tramée en vue de l'économie de la construction, celle-ci fait appel à des techniques de pointe - notamment large usage du béton précontraint et conditionnement général - grâce auxquelles l'architecte et son équipe ont tenté de donner à ce bâtiment un grand caractère ${ }^{34}$. 


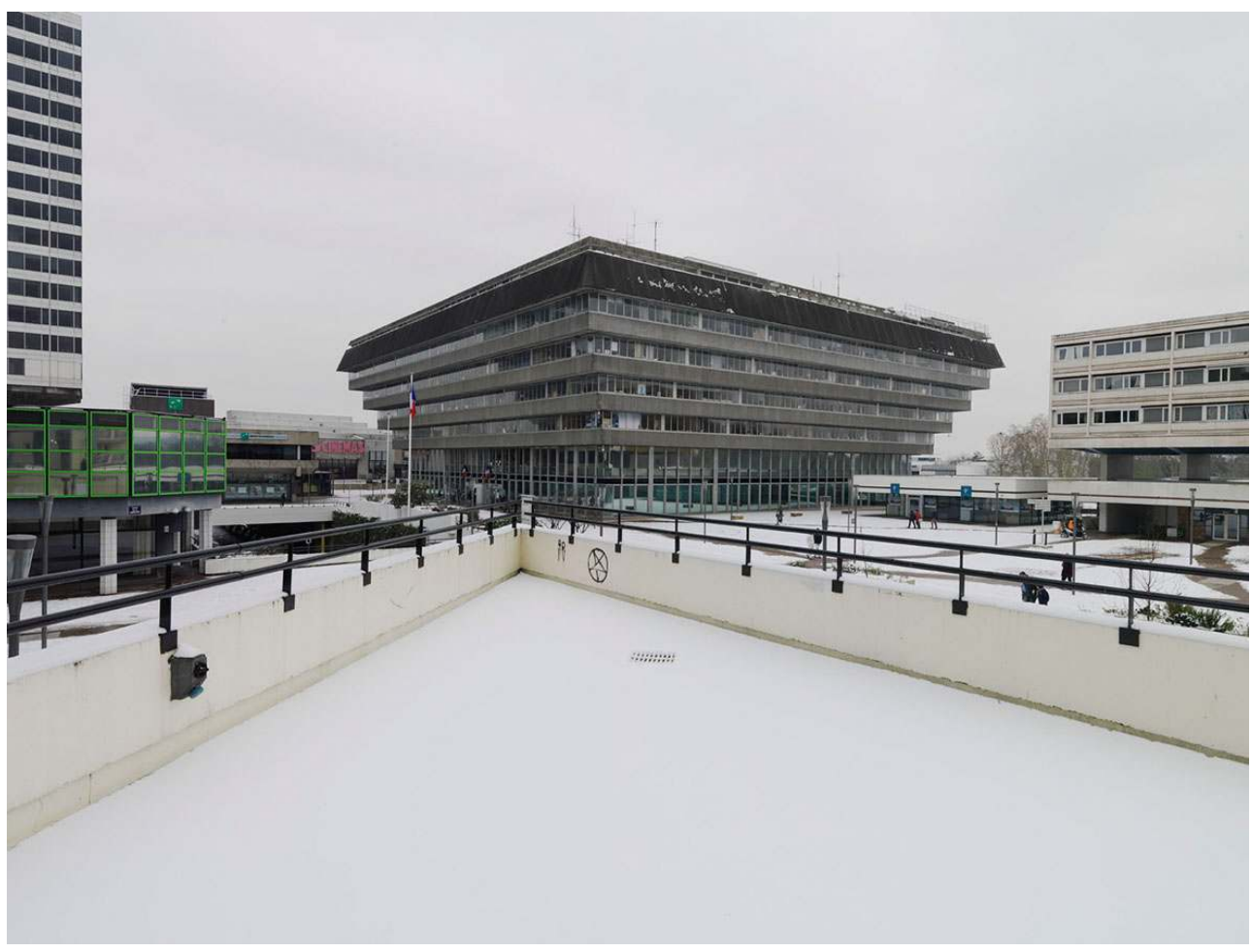

La préfecture depuis le parvis.

Phot. Laurent Kruszyk. @ Région île-de-France/Laurent Kruszyk/ADAGP 2015.

21 Mais ce "grand caractère » (fig. 12) que possède l'édifice fut à l'origine de tensions avec la maitrise d'ouvrage, comme en témoigna Bernard Hirsch :

André Chadeau - et je partage cet avis - aurait voulu une préfecture blanche. Nous sentons Henry Bernard réticent. Son idée est de faire un revêtement en «choc béton ». Devant nos incertitudes, il nous emmène visiter un immeuble construit à Paris avec ce procédé. La façade est blanche comme celle d'une cathédrale ravalée par Malraux. C'est exactement ce que nous souhaitons... Quelques mois plus tard, nous voyons arriver sur le chantier les panneaux de revêtement, du gris le plus triste que l'on puisse imaginer, imitant à s'y méprendre la couleur du béton. Le Préfet s'insurge - « Mais vous m'avez donné votre accord, répond l'architecte, nous avons même visité ensemble un chantier témoin. - Je vous ai donné accord pour le choc béton, à condition qu'il soit blanc. - Je suis désolé. Il y a un malentendu. Malheureusement il est trop tard pour changer, tous les panneaux sont déjà fabriqués $\aleph^{35}$.

\section{L'intervention de Joseph-André Motte et de François Stahly}

22 Henry Bernard attacha un soin tout particulier à l'esthétique de son édifice, aussi bien pour son volume que pour ses matériaux - aluminium des huisseries, panneaux d'amiante-ciment émaillé qui habillent les sous-faces extérieures des planchers, claustras préfabriqués qui décorent le premier niveau du hall (fig. 13), etc. Il fit également intervenir le sculpteur François Stahly (1911-2006) ${ }^{36}$ et le décorateur Joseph-André Motte $(1925-2013)^{37}$ avec lesquels il avait collaboré lors de l'édification de la Maison de la Radio ${ }^{38}$ 


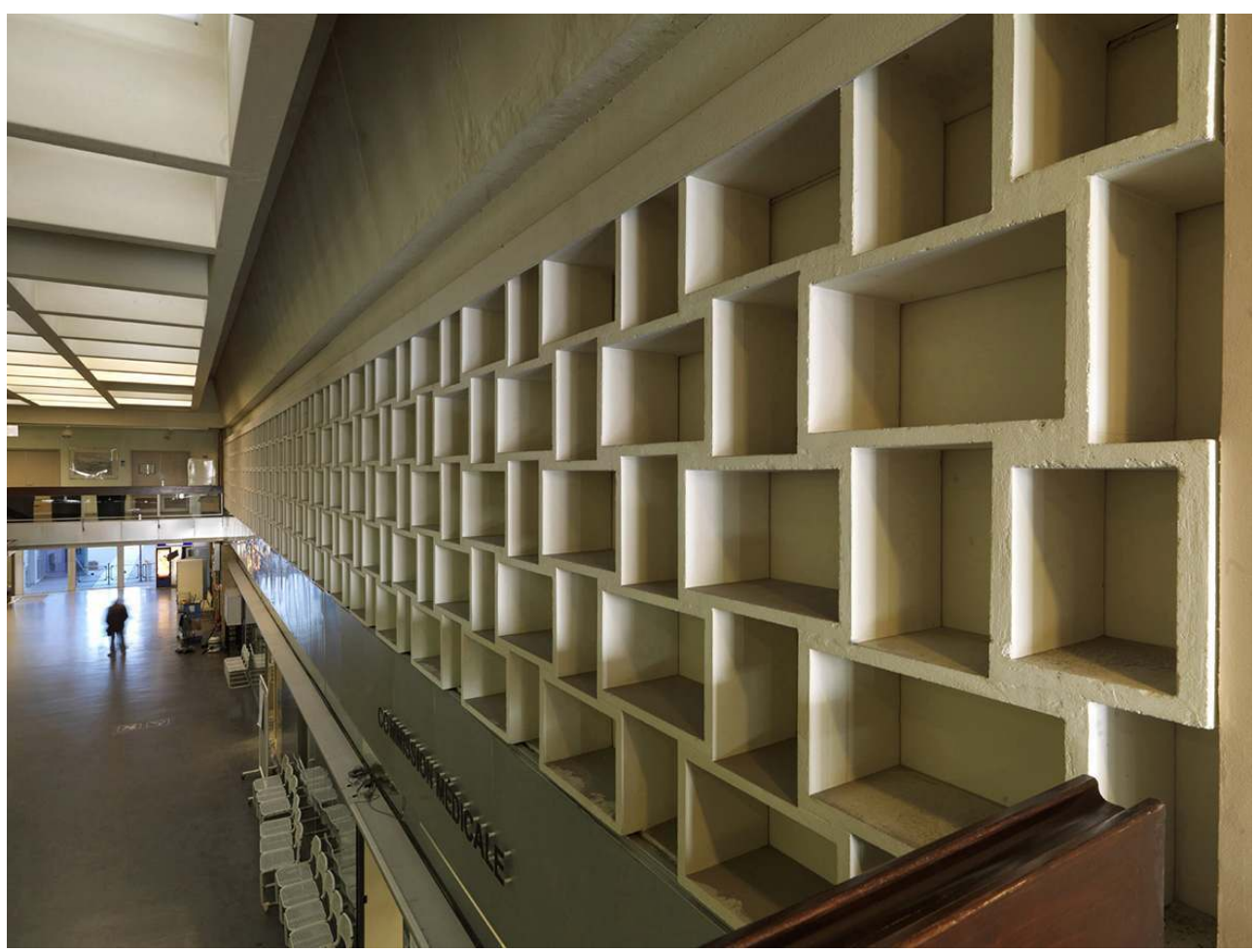

Claustras préfabriqués du premier niveau du hall dont les jours furent par la suite occultés.

Phot. Laurent Kruszyk. (c) Région Île-de-France/Laurent Kruszyk/ADAGP 2015.

François Stahly traita les bétons apparents et réalisa notamment, avec son atelier de Meudon, une sculpture de parement qui habille les quatre piles porteuses du toit du hall. Les agrégats ocre choisis par le sculpteur permirent, comme le souligna Bernard, de redonner «à ces structures massives un aspect moins brutal ${ }^{39}$ ". Joseph-André Motte fut quant à lui chargé « par l'architecte de cette tâche passionnante qui va de l'aménagement et des décors à l'invention des meubles ${ }^{40}$. Il réalisa, entre autres, le grand salon d'honneur ainsi que la salle des délibérations du conseil général, deux espaces qui furent ensuite totalement remaniés et dont aucun aménagement de Motte ne subsiste.

D'une surface de 300 mètres carrés, le grand salon (fig. 14) avait un sol habillé de plaques carrées d'acier inoxydable de 45 centimètres - selon la trame de l'édifice de 1,80 mètre. Ce revêtement, réputé non rayable, silencieux et " inaltérable à la cigarette, à la poussière ", apparaissait idéal pour une «forte densité de population peu soucieuse du sol qu'elle piétine $^{41} »$. Sur ces dalles étaient disposés de vastes et épais tapis de laine écrue sur lesquels étaient placés de grands fauteuils garnis de mousse et revêtus de tissu orange vif. Étant situé en mezzanine au premier étage du hall, le grand salon était isolé par douze panneaux amovibles « en épis » qui pouvaient devenir un mur de séparation, tandis que les parements de béton du sculpteur Stahly agrémentaient les murs. Enfin, cette vaste pièce s'ouvrait au sud-ouest sur le parc urbain grâce à un grand mur vitré. 
Figure 14

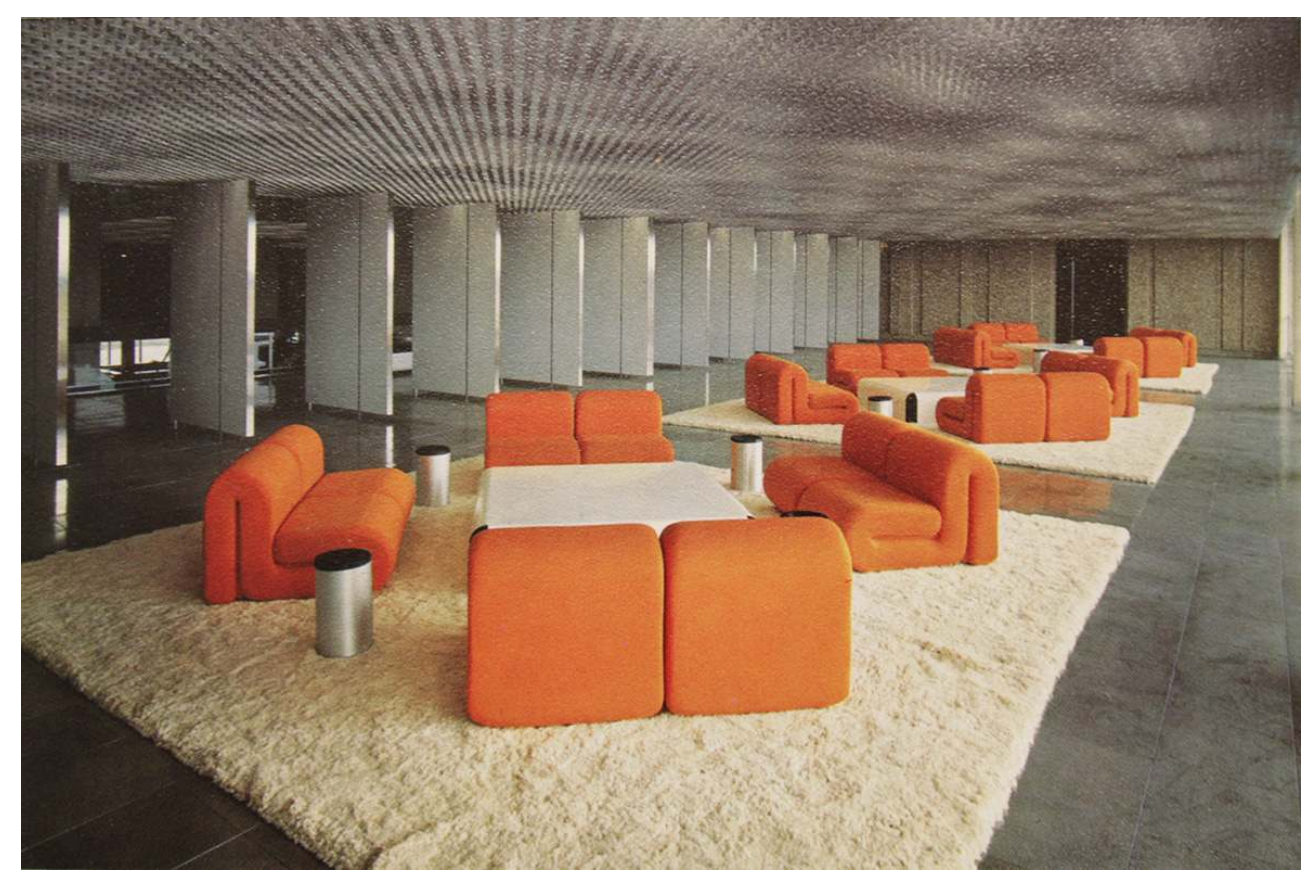

Le grand salon aménagé par Joseph-André Motte, vers 1970 (archives de l'IFA ; fonds Bernard 266 AA).

Repro. Léo Noyer-Duplaix. (C) Académie d'architecture/Cité de l'architecture et du patrimoine/Archives d'architecture du Xxe siècle/ADAGP.

D’une surface de 250 mètres carrés, la salle des délibérations du conseil général (fig. 15) abritait quant à elle une grande table carrée de 6 mètres composée d'un plateau en dalles d'acier inoxydable. Sur celui-ci, 36 pupitres intégraient un «bas parleur », un cendrier et une niche à document. En outre, la partie supérieure du plateau cachait des volets escamotables automatiques commandés par le président du conseil. Par l'action d'un simple bouton, ce dernier pouvait faire lever l'ensemble des éléments qui révélaient alors le micro et les boutons d'appel et de réception de chaque conseiller. Composée d'un ensemble de 250 pièces montées sur place, cette table reposait sur une moquette en laine écrue et était éclairée par des rampes fluorescentes dissimulées par des grandes lames d'acier inoxydable qui formaient de grands carrés successifs. Pour Motte, «le métal seul permettait cette construction dans cette rigueur de finition ", l'ambiance étant « digne, sans austérité, nette et précise, propice au travail de groupe organisée ${ }^{42}$ ». 


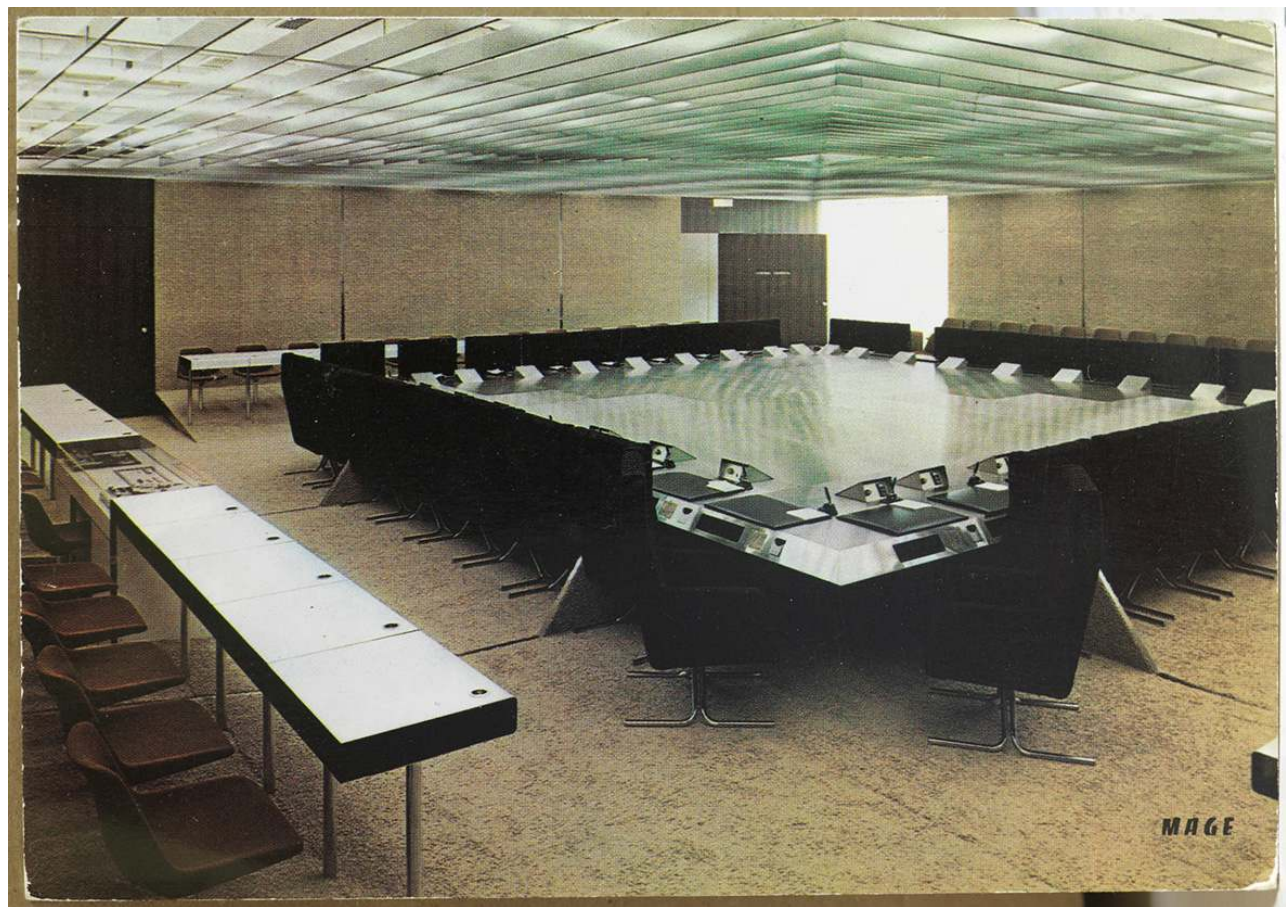

La salle des délibérations du conseil général aménagée par Joseph-André Motte, carte postale, vers 1970 (archives de la préfecture du Val d'Oise).

Repro. Laurent Kruszyk. ( ) Région Île-de-France/Laurent Kruszyk/ADAGP 2015.

Joseph-André Motte conçut également le bureau du préfet (fig. 16) ainsi que sa salle de conférence (fig. 17). Ces deux pièces étaient habillées d'un sol en tapis de laine écrue, sur lequel prenaient place des meubles édités par le Mobilier national et dont les premiers prototypes furent exposés au $47^{\mathrm{e}}$ Salon des artistes décorateurs, en 1967, dont le thème était «l'Art de vivre». Se détachant de la moquette et du cuir des fauteuils, la table de conférence - de 6 par 1,20 mètres -, le bureau du préfet ${ }^{43}$ et sa table basse ${ }^{44}$ sont plaqués d'acier inoxydable, leurs chants étant en ébène de macassar. Comme le souligna Motte, cet ameublement - dont le bureau et la table basse sont encore utilisés par le préfet soutenait « la confrontation avec les bétons Stahly » qui habillaient les murs ${ }^{45}$. 
Figure 16

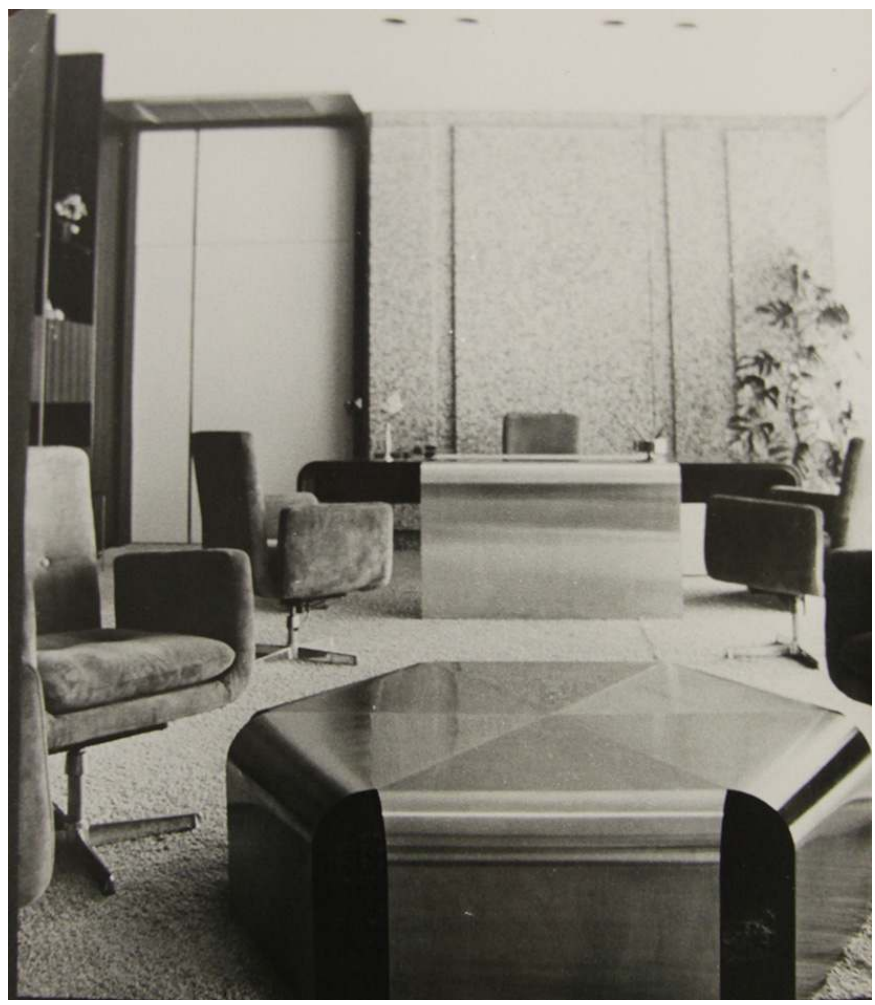

Le bureau du préfet aménagé par Joseph-André Motte, vers 1970 (archives de l'IFA ; fonds Bernard 266 AA).

Repro. Léo Noyer-Duplaix. (C) Académie d'architecture/Cité de l'architecture et du patrimoine/Archives d'architecture du xxe siècle/ADAGP. 
Figure 17

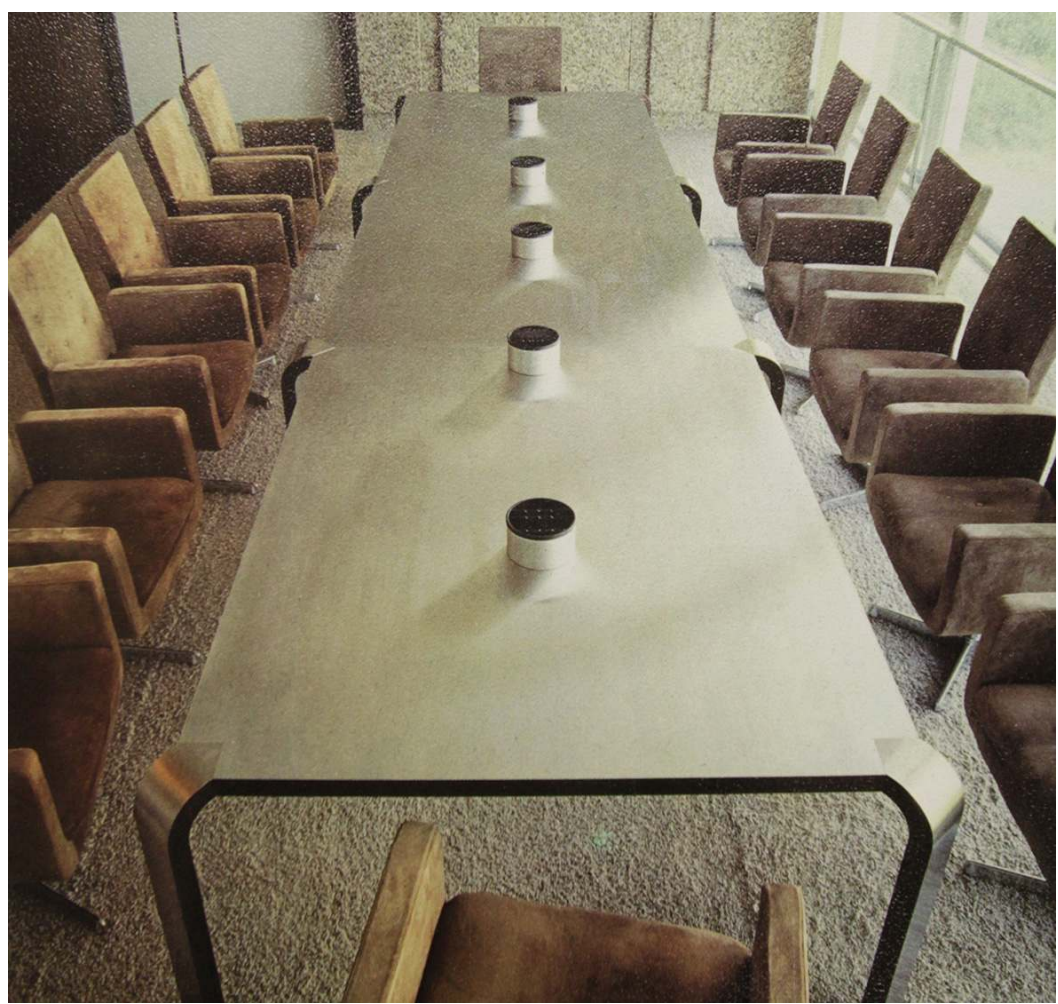

La salle de conférences du préfet aménagée par Joseph-André Motte, vers 1970 (archives de l'IFA ; fonds Bernard $266 \mathrm{AA}$ ).

Repro. Léo Noyer-Duplaix. (C) Académie d'architecture/Cité de l'architecture et du patrimoine/Archives d'architecture du Xxe siècle/ADAGP.

\section{Au-delà du monument ?}

\section{La « préfecture-drugstore »}

En 1967, Max Querrien, alors directeur de l'Architecture au ministère des Affaires culturelles, publia une tribune dans L'Architecture d'aujourd'hui dans laquelle il posait les principes d'une nouvelle architecture administrative :

Pour construire une préfecture, une cité administrative, un hôtel de ville, une maison de la culture, une maison de jeunes, une école d'architecture, une faculté, un hôpital, il ne suffit pas de savoir combien de mètres carrés de planchers à destinations diverses on est invité à « fournir » à l'issue d'un travail de bureau qui ne repose ni sur l'analyse de l'expérience d'hier, ni sur l'exploitation prospective des mutations en puissance, ni sur l'épaisseur sociale des prises de conscience partagées. Ce qui compte avant tout, c'est la signification globale de l'œuvre, c'està-dire la nature et le contenu du message que les hommes capteront en pénétrant dans une certaine structure d'espace. Pour prendre un exemple élémentaire, une nouvelle préfecture doit-elle être conçue comme un reflet de la tradition napoléonienne avec la composition "monumentale", la façade souveraine et le mobilier Empire? Ou comme une forteresse abritant des fichiers, des recueils de textes, des tampons, des ordinateurs et accessoirement quelques «servants de pièces " pour manipuler tout cela ? Ou comme un milieu de travail, de rencontre, de 
confrontation, de dialogue, de mise en commun, offert non seulement aux fonctionnaires mais aussi aux élus et même aux citoyens ? ${ }^{46}$ « en dialogue » avec les administrés. La construction de l'édifice cergypontain fut alors l'occasion de mettre en œuvre ces nouveaux principes d'architecture administrative. Henry Bernard souligna ainsi que "à la différence des solutions traditionnelles bâtiments hermétiquement clos au fond d'une place - la préfecture du Val-d'Oise est une maison de verre transparente et accessible en permanence $»^{47}$. Elle fut dès lors imaginée comme une «maison accueillante» dont «l'accessibilité pour tous était la première volonté affirmée par l'édifice $»^{48}$. Au cœur de ce dispositif, le grand hall, espace unique d'accueil du public destiné à centraliser toutes les formalités mais également à accueillir d'autres activités. Le "caractère de service public» était en effet "confirmé par l'adjonction d'équipements tels que bureau de poste, agence de voyage, SNCF, Air France, location de théatres, régie des tabacs » tandis que la corbeille devait devenir le « hall d'exposition de la ville nouvelle en gestation ${ }^{49}$ ». Ce vaste hall mêlant guichets de démarches administratives et services et commerces du quotidien fut à l'origine, comme le rappella Bernard Hirsch, du surnom par la presse de "préfecture-drugstore ${ }^{50}$ ». Cette incursion de la quotidienneté, du profane, dans l'espace sacré de la République - à replacer dans le contexte d'un édifice construit en plein champ, sans aucun service ni équipement alentour - fut l'aboutissement de la volonté du préfet délégué André Chardeau "d'abattre les barrières entre l'Administration et les usagers ${ }^{51}$ ». Mais ce dessein de dépassement du cadre administratif fut-il réellement partagé par Bernard ?

Hirsch évoqua les «idées très arrêtées » de l'architecte, s'agissant de la symbolique d'une préfecture $^{52}$. L'autorité de l'État devait en effet pour Bernard se manifester dans la noblesse, le recueillement et la solennité, en somme dans une gravitas qu'était loin d'incarner une « préfecture-drugstore ». Cette différence de points de vue fut à nouveau à l'origine de tensions comme en témoigna Bernard Hirsch à propos de l'installation d'un restaurant dans la corbeille du grand hall :

Dans ce vaste espace, situé au fond de la Préfecture et donnant directement sur le parc, André Chadeau avait décidé d'aménager un restaurant. Cette idée ne plaisait manifestement à l'architecte qui ne souhaite pas mêler des activités profanes à l'exercice austère du pouvoir que représentait à ses yeux une Préfecture. Mais Henry Bernard ne cherche pas à entrer en conflit direct avec le Préfet. Ce n'est que beaucoup plus tard, au moment où le restaurateur se préoccupera d'installer ses fourneaux qu'il découvrira que la ventilation est insuffisante et que l'odeur de graillon risque de se répandre dans tous les bureaux. Il faut renoncer à utiliser la corbeille et, au dernier moment, improviser l'aménagement d'un restaurant et de quelques magasins à l'extérieur de la Préfecture, sous la dalle, à un emplacement où tout éclairage naturel est impossible ${ }^{53}$.

Le restaurant fut donc finalement installé hors de l'édifice, sous la dalle, dans un espace qui accueillit le drugstore. Subsistent toutefois, au rez-de-jardin de la préfecture, les vastes cuisines (fig. 18) du restaurant jamais mis en service, témoins d'une volonté de dépasser le cadre administratif qui ne fut que de courte durée. 
Figure 18

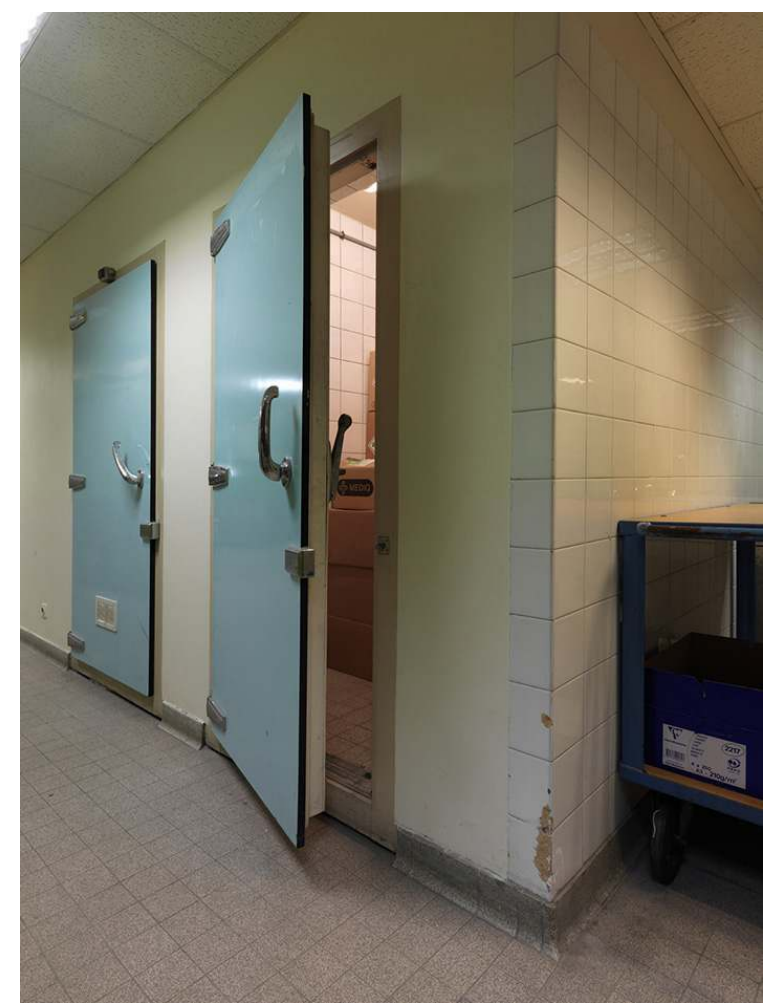

Chambres froides des cuisines du restaurant jamais mis en service.

Phot. Laurent Kruszyk. @ Région île-de-France/Laurent Kruszyk/ADAGP 2015.

\section{Un édifice en frange de dalle}

31 Loin de se limiter à l'organisation des espaces intérieurs, ce «nouveau type d'intervention de l'administration auprès des citoyens » qu'incarna la préfecture cergypontaine se manifesta également par l'implantation et le volume de l'édifice. Première réalisation d'ampleur de la ville nouvelle, elle devait symboliser le parti d'aménagement du centre de l'agglomération. Et, afin "d'assurer une fluidité satisfaisante pour la circulation automobile générée par la forte densité du programme général ", « d'économiser les espaces au sol » en « jouant avec les superpositions plutôt qu'avec les juxtapositions » et « d'avoir des plates-formes d'échanges optimum » entre les différents modes de déplacement, le quartier de Cergy-Préfecture fut urbanisé sur dalle (fig. 19) ${ }^{5455}$. 


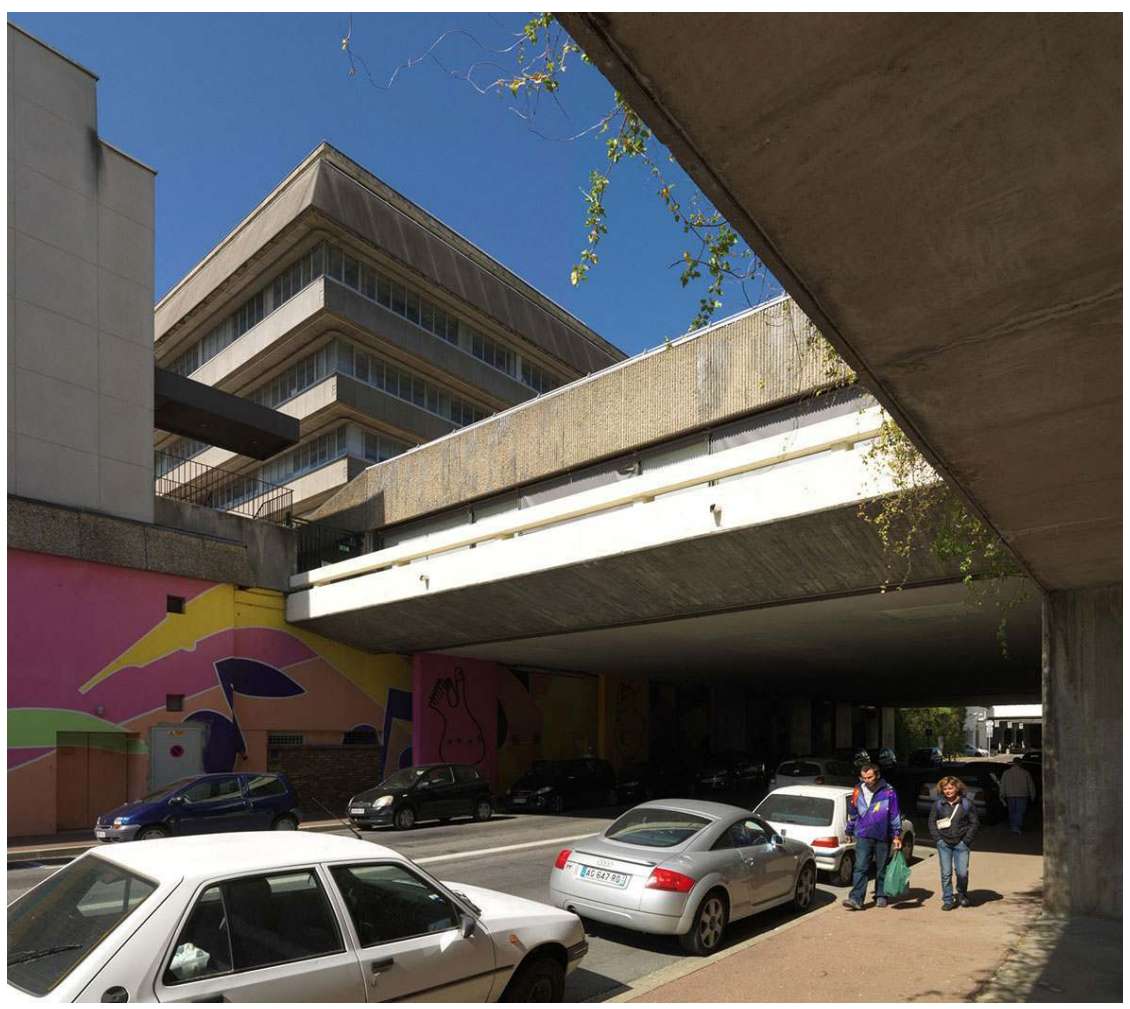

La dalle et la préfecture depuis le niveau de circulation automobile.

Phot. Laurent Kruszyk. @ Région île-de-France/Laurent Kruszyk/ADAGP 2015.

L'histoire de son édification peut être divisée en deux étapes: 1970-1975, qui vit la construction simultanée des îlots sud-ouest et nord-est, et les années 1980, qui réunirent ces deux entités lors de l'opération dite "centre-gare ». Durant la première période d'aménagement, celle amorcée par la construction de la préfecture, le fonctionnalisme fut prééminent. La seconde période marqua quant à elle la volonté de renouer avec des aménagements traditionnels afin de rendre le quartier " plus humain ». Les avenues, rues et places furent redécouvertes. Si le principe de séparation des circulations fut maintenu, il fut toutefois aménagé différemment. Les voies de circulation ne furent plus simplement juxtaposées mais hermétiquement séparées, de manière à ce que la dalle ne soit plus perceptible. Deux espaces de part et d'autre du centre culturel et administratif AndréMalraux ${ }^{56}$ témoignent aujourd'hui de ces deux époques d'aménagement (fig. 20). Au nord-est, la Grand'Place, avec son dallage et ses platanes, fait référence aux centres anciens, dans une approche qui peut être qualifiée de post-moderne. À l'inverse, au sudest, le parvis de la préfecture, minéral et dépouillé, caractérise le fonctionnalisme des débuts de la ville nouvelle. La préfecture en est aujourd'hui le symbole. De plus, sa situation au sein du quartier reste on ne peut plus importante car, implantée en frange de dalle, elle en assure l'accès depuis le parc urbain. 


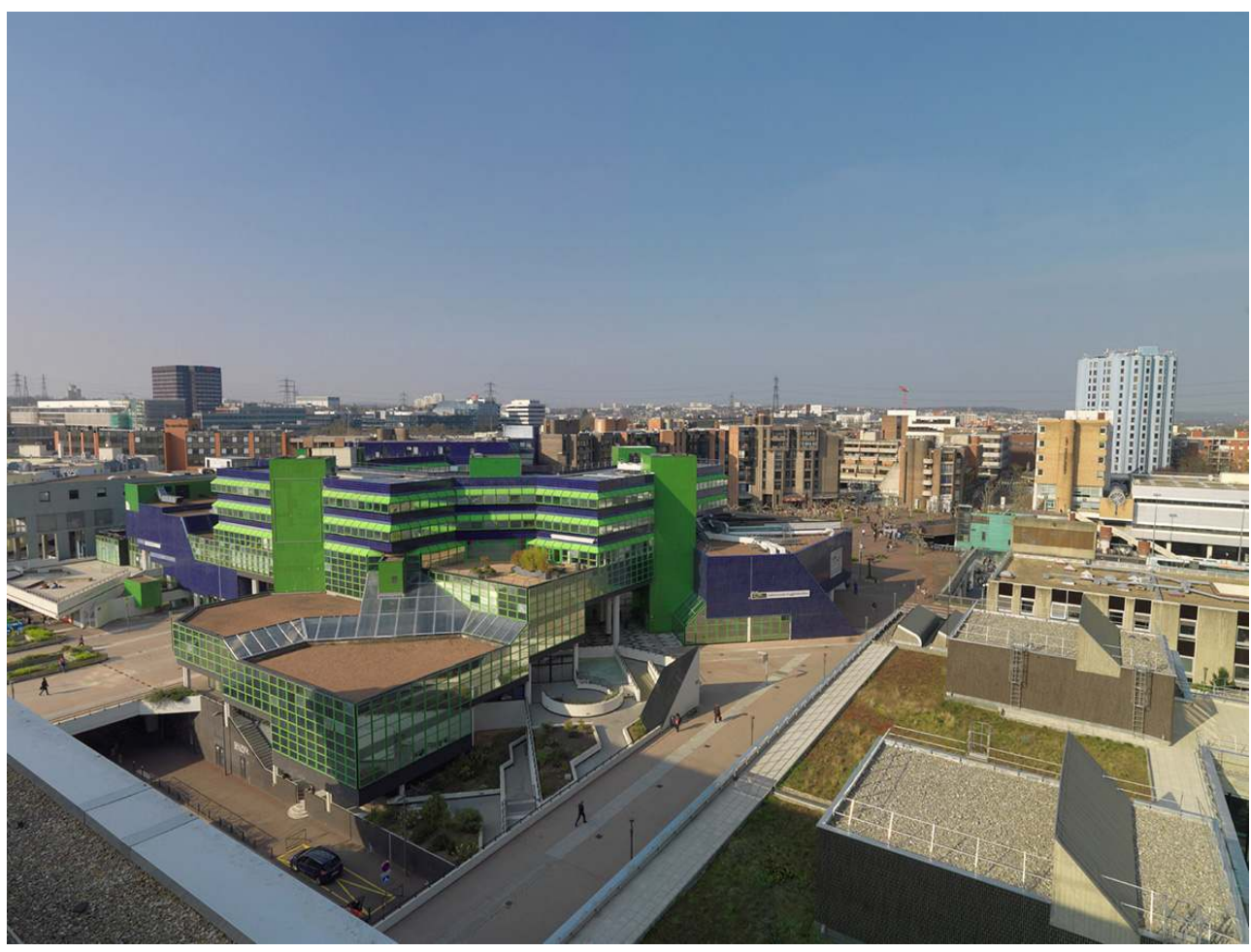

Depuis le toit de la préfecture, au premier plan le centre culturel et administratif André Malraux, au second la Grand'Place.

Phot. Laurent Kruszyk. @ Région Île-de-France/Laurent Kruszyk/ADAGP 2015.

Dans les premiers projets d'Henry Bernard, la corbeille de l'édifice située en rez-de-jardin était aveugle. La maîtrise d'ouvrage lui demanda d'ouvrir cet espace par une large façade vitrée sur le parc. Avec ses deux rez-de-chaussée - un au niveau de la dalle et l'autre au niveau du jardin - la préfecture devint alors un lieu de transition. Le cheminement du piéton se prolongeait en effet dans le hall qui constituait alors, comme le souligna Bernard, « un élément de place couverte » :

La Préfecture ne se présente plus comme naguère sous la forme d'une citadelle constituant le frontispice d'une immense place. L'édifice est implanté sur la place même, son rez-de-chaussée n'étant plus qu'un élément de place couverte prévu pour attirer et accueillir le piéton. Sa transparence complète permet également des perspectives sur la ville et le parc ${ }^{57}$.

Le vaste hall fut ainsi au cœur du dispositif de dépassement du cadre administratif. Abritant aussi bien des guichets destinés aux formalités que des services du quotidien, il devait tenir lieu de place couverte, lieu de circulation et d'échanges. Mais les motifs sécuritaires eurent raison de ce dessein, et la préfecture ne devint accessible au public que depuis la dalle. Ce bâtiment charnière, qui assurait une continuité, devint rupture (fig. 21). 


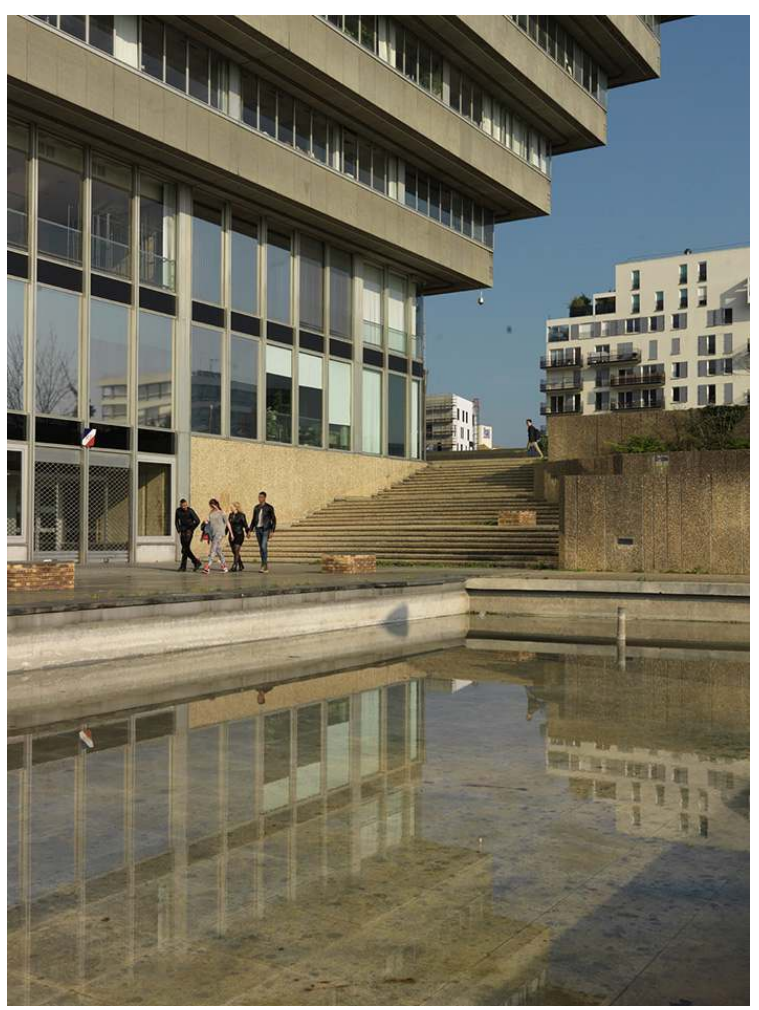

L'accès au « rez-de-jardin » de la préfecture depuis le parc.

Phot. Laurent Kruszyk. @ Région île-de-France/Laurent Kruszyk/ADAGP 2015.

\section{Un renversement des valeurs?}

Le volume de la préfecture du Val-d'Oise, qui forme une pyramide inversée, fut-il un avatar parmi d'autres de la tentative de redéfinition de l'architecture administrative? Bernard Hirsch, dans son témoignage sur l'aménagement de Cergy-Pontoise, souligna l'affiliation de l'édifice avec l'hôtel de ville de Boston (Kallmann McKinnell \& Knowles architectes, 1963-1968) (fig. 22) et sa monumentalité en rupture :

Les architectes de la Mission, qui - comme c'est la règle dans la profession - ne sont pas tendres pour leurs confrères, ne ménagent pas les critiques au projet d'Henry Bernard : style pompier, architecture-cendrier (il est vrai que pendant des années nous déposions nos mégots dans une petite maquette de la préfecture qui se prête admirablement à cet usage). Jean Coignet a une mine particulièrement réjouie le jour où il découvre qu'Henry Bernard s'est inspiré de l'hôtel de ville de Boston et il éprouve un malin plaisir à montrer que l'imitation a perdu tout ce qui fait le caractère du modèle.

Mon opinion est plus mesurée. Je trouve le projet coûteux et peu rationnel avec ses porte-à-faux successifs mais ce n'est pas mon rôle de défendre les finances de l'État ou de veiller au confort des fonctionnaires. En tant que responsable de la Ville nouvelle, j'apprécie que le premier édifice ait un caractère monumental et une silhouette originale, reconnaissable entre toutes qui marque une rupture avec les cubes ou les parallélépipèdes, vocabulaire habituel de l'architecture contemporaine 58 . 


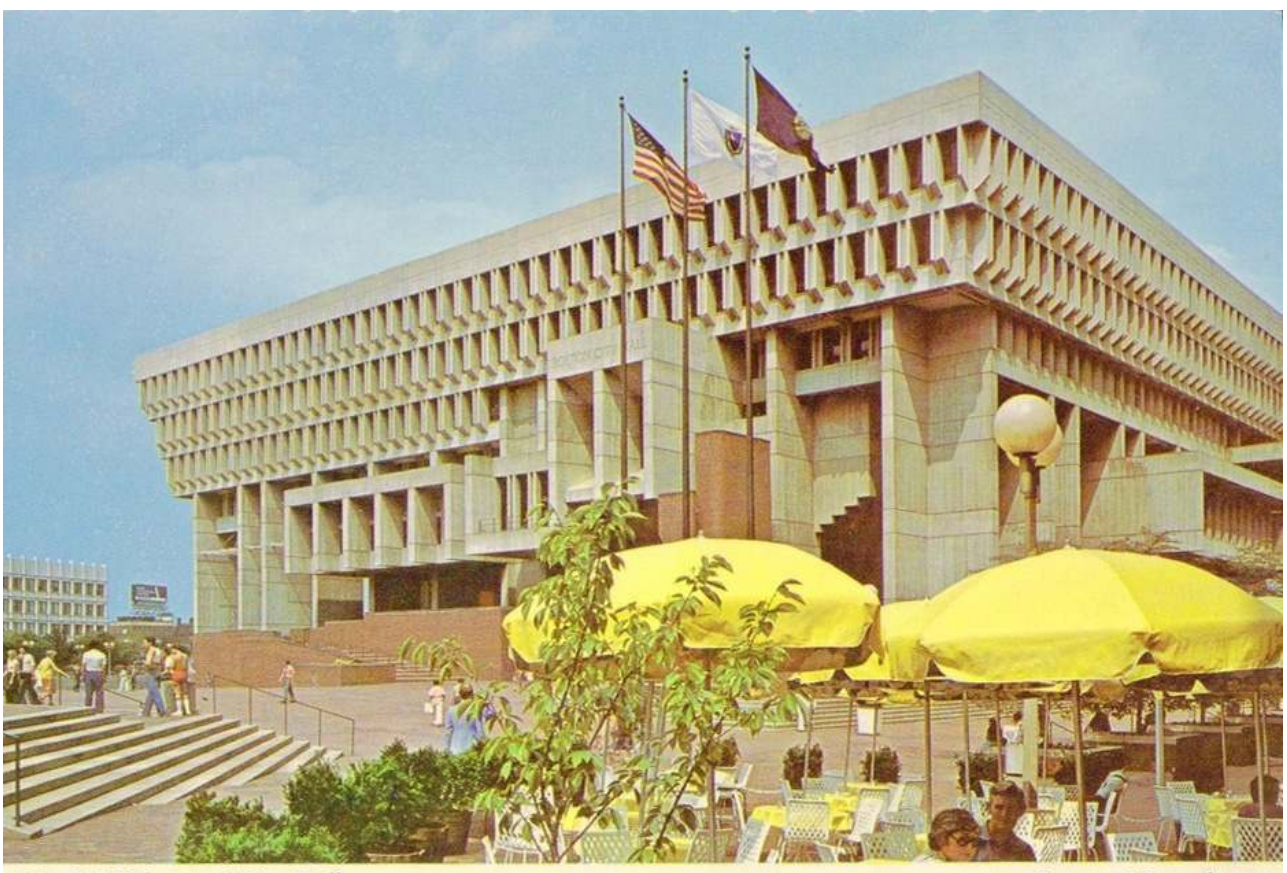

L'hôtel de ville de Boston, carte postale, vers 1980 (coll. part.).

Repro. Léo Noyer-Duplaix. @ Droits réservés.

Bien que souvent rapportée dans la littérature ${ }^{59}$, la référence au siège de la municipalité bostonienne ne semble jamais avoir été revendiquée par l'architecte. En outre, l'utilisation par Bernard de la pyramide ne constitua pas un cas isolé. Cette forme renversée ou non - fut employée à de nombreuses reprises dans l'architecture de la fin des Trente Glorieuses, comme le souligne François Loyer ${ }^{60}$. Elle fut ainsi utilisée lors de l'édification de l'hôtel de ville de Nanterre (Jean Darras et Yves Bedon, 1967-1974), du bâtiment administratif de la raffinerie Elf-Erap à Feyzin (Henri Vidal et Yves Jenkins, 1967), du siège social de Pernod à Créteil (Jean Willerval, 1974) (fig. 23), de la souspréfecture des Hauts-de-Seine à Boulogne-Billancourt (Fralen et Steinz, 1976) ou encore de la préfecture de Seine-Saint-Denis, à Bobigny, qui prend la forme d'une pyramide dissymétrique (Michel Folliasson, 1971). Loyer note ainsi que ces différentes réalisations « empruntent leur silhouette au projet de musée d'Art moderne de Caracas, dessiné en 1955 par Oscar Niemeyer ${ }^{61}$. 


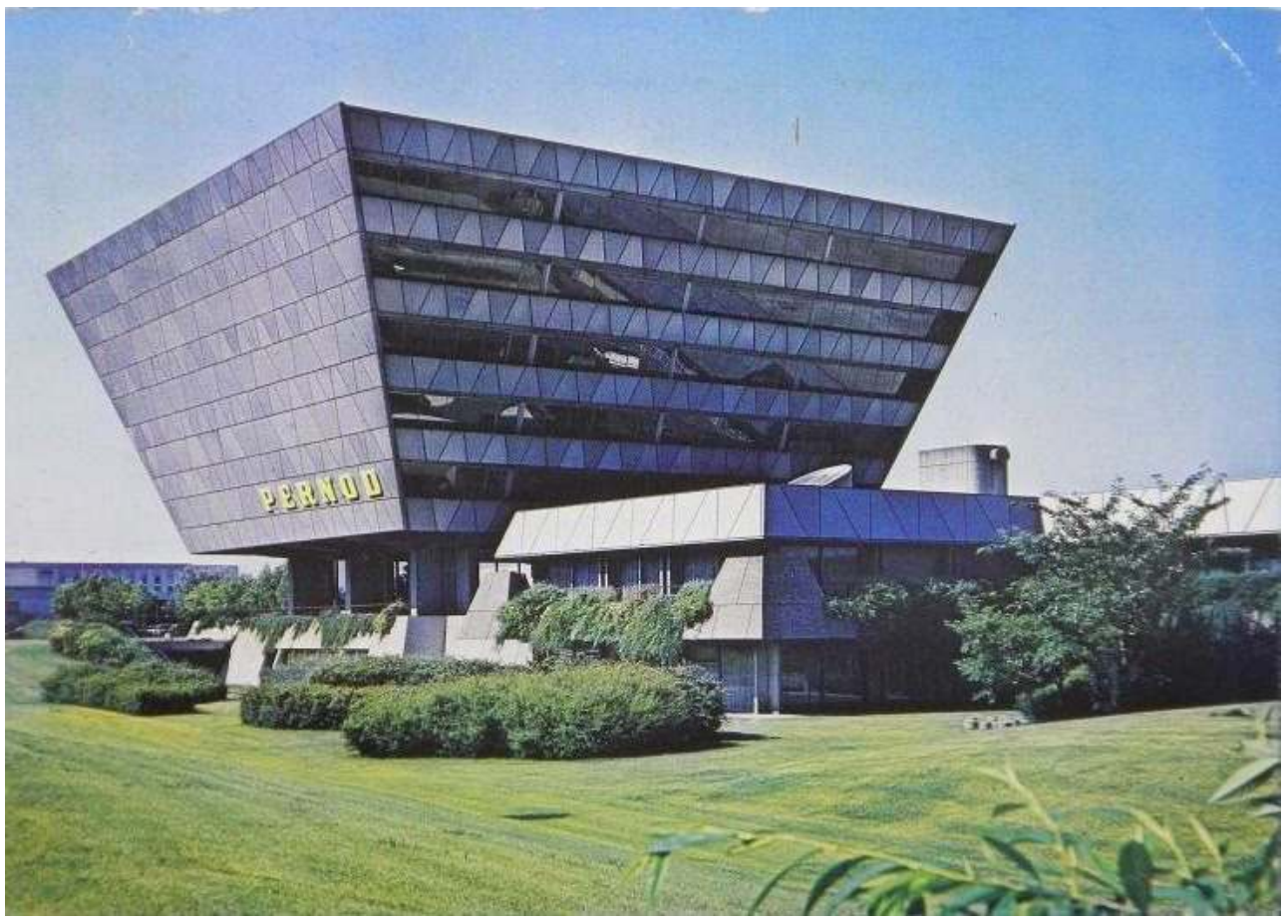

Siège social de Pernod à Créteil, carte postale, vers 1974 (coll. part.).

Repro. Léo Noyer-Duplaix. @ Droits réservés.

L'inversion de la pyramide par Henry Bernard fut-elle alors un renversement des valeurs? Comme le souligna Ionel Schein en 1971 dans L'Information d'histoire de l'art, l'architecture de la préfecture cergypontaine fut d'abord "contemporaine du temps historique de sa construction ». "Toute autre interprétation» était selon lui «vaine " ${ }^{62}$. Dans Bâtir, Georges Brégou rapporta quant à lui « l'expression architecturale inspirée de l'ancienne Égypte ${ }^{63} \%$. Plus récemment, Bertrand Lemoine, dans un article sur l'architecture des préfectures contemporaines, note que « la référence au pouvoir absolu des pharaons n'est sans doute pas délibérée » et « qu'il y a surtout la volonté de créer une forme bien identifiable et clairement lisible dans le chaos urbain que l'on anticipe déjà ${ }^{64}$. Les sources ne témoignant d'aucun propos de l'architecte, la symbolique renversée du pouvoir que pourrait incarner l'édifice reste incertaine mais peu probable. S'inscrivant dans le mouvement d'une époque, Bernard chercha en effet d'abord à conférer à l'édifice « un caractère architectural affirmé de bâtiment-pilote » ${ }^{65}$ que la forme d'une pyramide inversée lui attribua. Elle permit également, grâce au jeu de rapport entre les pleins et les vides, d'estomper l'aspect monolithique du bâtiment. Elle s'inscrivit enfin dans la quête de la monumentalité qui caractérise la carrière d'Henry Bernard, architecte qui n'eut de cesse de questionner l'identité monumentale. À Cergy-Pontoise, cette dernière eut la particularité de survenir "en plein champ ", sans positionnement à l'égard d'un tissu urbain préexistant. Cette singularité d'une création ex nihilo explique sûrement le jugement pour le moins catégorique et sévère que porte Gérard Monnier sur l'édifice : « si elle était emphatique lorsqu'elle surgissait isolée dans un espace libre, elle est devenue, maintenant qu'elle est enserrée parmi des bâtiments autrement plus bavards, un bâtiment insipide et peu lisible $»^{66}$. Loin d'être fade ou brouillonne, la préfecture du Vald'Oise doit être comprise eu égard à l'histoire de l'aménagement de la ville nouvelle et à 
la volonté affirmée de dépasser le cadre administratif. Renouvelant la typologie, elle permit, comme le rappelle Jean-Yves Andrieux, avec les autres préfectures des départements nouveaux, de donner "à la République une physionomie nettement identifiable, non plus par soumission à une syntaxe oubliée, mais par opposition à un paysage urbain disloqué $»^{67}$.

\section{NOTES}

1. - BERNARD, Henry. «Val d'Oise : une préfecture aux champs ». La Construction moderne, janvierfévrier $1970, \mathrm{n}^{\circ} 1$, p. 22.

2. - Voir sur le site : http://www.theses.fr/s79834 [consulté le 17/04/2018].

3. - Cette réorganisation du territoire francilien avait pour motivations de permettre une meilleure maitrise de l'expansion urbaine de la banlieue parisienne, de revitaliser certains territoires - s'agissant tout particulièrement de la Seine-et-Oise -, d'asseoir le District de Paris nouvellement créé en démembrant le département de la Seine qui aurait pu lui porter une concurrence politique, et de limiter l'influence du parti communiste français à la Seine-SaintDenis et au Val-de-Marne.

4. - HIRSCH, Bernard. L'Invention d'une ville nouvelle, Cergy-Pontoise, 1965-1975, récit d'un témoin. Paris : Presses de l'École nationale des ponts et chaussées, 1990, p. 34.

5. - Ces terrains furent dans un premier temps nommés « quartier de la boucle de l'Oise » avant de prendre le nom de « quartier de la Préfecture ». Par la suite dénommé « Cergy-Préfecture », le quartier porte aujourd'hui le nom de « Grand Centre ».

6. - Ancien élève de l'École polytechnique et de l'École nationale des ponts et chaussées, Bernard Hirsch fut directeur des Travaux publics de Mauritanie et du Mali, chargé de mission à la direction des Constructions scolaires au ministère de l'Éducation avant d'être nommé ingénieur des Ponts et Chaussées à Versailles. Depuis ce poste, il commença à travailler à l'aménagement de la ville nouvelle de Cergy-Pontoise, dont il fut nommé le 18 juillet 1966 « directeur responsable ». Prenant la tête de l'établissement public d'aménagement de la ville lors de sa création en 1969, il le dirigea jusqu'en 1975, date à laquelle il devint directeur régional de l'Équipement de la région Île-de-France. En 1983 il fut nommé directeur de l'École nationale des ponts et chaussées, poste qu'il occupa jusqu'à sa mort. En 1990 furent publiées à titre posthume ses mémoires (voir note 3) portant sur l'aménagement de Cergy-Pontoise, témoignage précieux d'un acteur majeur du projet.

7. - Urbaniste et architecte DPLG, Jean Coignet participa à l'aménagement de la ville nouvelle jusqu'en 1969 en tant que chargé de mission à l'Institut d'aménagement et d'urbanisme de la région parisienne (IAURP). Par la suite, il s'établit à son compte et participa notamment à des projets de revalorisation de quartiers anciens de villes du sud de la France.

8. - Diplômé de l'École des beaux-arts, premier second grand prix de Rome en 1956, architecte en chef des Bâtiments civils et Palais nationaux, Michel Folliasson fut, entre autres, l'auteur en 1971 du centre administratif et de la préfecture de la Seine-Saint-Denis à Bobigny.

9. - HIRSCH, Bernard. Op. cit., p. 78.

10. - La figure de Paul Bigot, « patron » d'Henry Bernard, selon l'expression consacrée à l'École des beaux-arts, fut prépondérante, spécialement à propos de la «monumentalité ». À sa mort, 
Bernard transféra sa monumentale maquette de Rome à l'université de Caen et veilla au dépôt de ses archives à l'Académie d'architecture.

11. - Voir dans la base Mérimée : notice PA14000102.

12. - Voir dans la base Mérimée : notice PA14000061.

13. - NOYER-DUPLAIX, Léo. " "L'hôpital-paquebot" d'Henry Bernard », In Situ [en ligne], 2017, n - 31, mis en ligne le 21 février 2017, < http://journals.openedition.org/insitu/13998 > [date de consultation 09/02/18].

14. - LOYER, François. Histoire de l'architecture française, de la Révolution à nos jours. Paris : Mengès/ Éd. du Patrimoine, 2006, p. 457.

15. - Il signa tout d'abord ses papiers « Eupalinos ", en référence à Eupalinos ou l'Architecte de Paul Valéry (1921) dont Bernard admirait l'œuvre. Par la suite l'architecte abandonna ce pseudonyme et utilisa son vrai nom.

16. - HIRSCH, Bernard. Op. cit., p. 54.

17. - Ibid., p. 55.

18. - Ibid.

19. - BERNARD, Henry. «Val d'Oise : une préfecture aux champs ». Art. cit., p. 22.

20. - BERNARD, Henry. Note non datée, p. 4 (Archives de l'IFA ; 266 AA 1/1).

21. - « La nouvelle Préfecture ». Bulletin d'information de la préfecture du Val d'Oise, juin 1970, nº 15, p. 6.

22. - BRÉGOU, Georges. « Préfecture en béton et verre à Cergy-Pontoise ». Bâtir, 6 octobre 1969, nº 178, p. 38.

23. - Ibid.

24. - BERNARD, Henry. «La nouvelle préfecture du Val d'Oise », s.n. et s.d., p. 16 (Archives de l'IFA ; 266 AA 11/4).

25. - HIRSCH, Bernard. Op. cit., p. 67.

26. - PLUM, Gilles. «Inventaire des préfectures et sous-préfectures ». Monuments historiques, décembre 1991, nº 178, p. 133.

27. - HIRSCH, Bernard. Op. cit., p. 290.

28. - BERNARD, H. « La nouvelle préfecture du Val d'Oise ». Art. cit., p. 17.

29. - Ibid.

30. - Ibid.

31. - BERNARD, Henry. «La préfecture du Val d'Oise ». Techniques et Architecture, novembre 1970, n 5, p. 60 .

32. - Voir dans la base Mérimée : notice PA00080174.

33. - BERNARD, Henry. « La préfecture du Val d'Oise ». Art. cit., p. 60.

34. - Ibid.

35. - HIRSCH, Bernard. Op. cit., p. 177-178.

36. - Actif en France et aux États-Unis, célèbre pour son art urbain monumental, François Stahly fut entre autres l'auteur de Le Labyrinthe ou La Forêt pétrifiée pour la faculté de Jussieu à Paris en 1965-1968, de Jardin labyrinthique pour l'Empire State Plaza du Capitole de l'État de New York, à Albany (États-Unis) en 1969-1974, ou encore de la cheminée du Front-de-Seine (1971, Paris, $15^{\text {e }}$ arrt.). En 1995, il fut élu membre de l'Académie des beaux-arts.

37. - Diplômé en 1948 de l'École des arts appliqués à l'industrie - aujourd'hui École nationale supérieure des arts appliqués et des métiers d'art - Joseph-André Motte travailla sur de nombreuses grandes commandes publiques. Il fut notamment à l'origine de l'aménagement intérieur de l'aéroport d'Orly en 1958-1961 ou encore de stations du métro parisien avec l'architecte Paul Andreu (1938-) dans les années 1970.

38. - François Stahly réalisa la sculpture Portiques ou L'Écho de la Forêt installée dans le grand hall de la Maison de la Radio.

39. - BERNARD, H. « La nouvelle préfecture du Val d'Oise ». Art. cit., p. 18. 
40. - BRÉGOU, G. « Préfecture en béton et verre à Cergy-Pontoise ». Art. cit., p. 38.

41. - MOTTE, Joseph-André. Brochure «L'acier inoxydable dans les aménagements intérieurs de la nouvelle préfecture du Val d'Oise à Cergy Pontoise, ou les raisons d'un choix ", s. d., société Uginox (Archives de l'IFA ; 266 AA 11/4).

42. - Ibid.

43. - Hauteur $0,75 \mathrm{~m}$, longueur $2,30 \mathrm{~m}$, profondeur $0,80 \mathrm{~m}$. Déposé le 7 juillet 1970 , référence GME 14454, numéro de dépôt 84.

44. - Hauteur 0,37 m, longueur et profondeur 1,12 m. Déposée le 7 juillet 1970, référence GME 14455, numéro de dépôt 84.

45. - MOTTE, Joseph-André. Op. cit.

46. - QUERRIEN, Max. "Sachons construire pour après-demain ». L'Architecture d'aujourd'hui, décembre $1967, \mathrm{n}^{\circ} 135$.

47. - BERNARD, H. « La préfecture du Val d'Oise ». Art. cit., p. 60.

48. - BERNARD, Henry. «Préfecture du Val d'Oise, propos de l'architecte », tapuscrit, avril 1988 (archives de la Préfecture du Val d'Oise).

49. - BERNARD, H. « La préfecture du Val d'Oise ». Art. cit., p. 60.

50. - HIRSCH, Bernard. Op. cit., p. 55.

51. - Ibid.

52. - Ibid., p. 54

53. - Ibid., p. 177-178.

54. - L'Urbanisme de dalles: actes du colloque des Ateliers d'été 1993 de Cergy-Pontoise. Paris/CergyPontoise: Presses de l'École nationale des ponts et chaussées/Établissement public d'aménagement de Cergy-Pontoise, 1995, p. 79.

55. - La dalle de Cergy-Préfecture est délimitée par les boulevards du Port et de l'Hautil, par l'A 15 ainsi que par le parc de la préfecture.

56. - Inauguré le 8 octobre 1979 par Jean-Philippe Lecat, ministre de la Culture et de la Communication, le centre culturel et administratif André-Malraux fut imaginé par Claude Vasconi (1940-2009) et Georges Pencreac'h (1941-).

57. - BERNARD, H. « La nouvelle préfecture du Val d'Oise », Art. cit, p. 16-17.

58. - HIRSCH, Bernard. Op. cit., p. 82.

59. - DUPAVILLON, Christian. "Architecture officielle : la façade des institutions ". L'Architecture d'aujourd'hui, avril 1980, $\mathrm{n}^{\circ} 208$, avril 1980, p. 45. Institut français d'architecture. Cergy-Pontoise: vingt ans d'aménagement de la ville, 1969-1989. Paris : Éditions Moniteur Images, 1989, p. 85. SAINTPIERRE, Caroline de. La Fabrication plurielle de la ville : décideurs et citadins à Cergy-Pontoise, 1990-2000. Paris : Creaphis, 2002, p. 48.

60. - LOYER, François. Op. cit., p. 464.

61. - Ibid.

62. - SCHEIN, Ionel. « Paris Construit ou d'une exposition qui l'est insuffisamment ». L'Information d'histoire de l'art, novembre-décembre 1971, nº 5, volume 16, p. 229.

63. - BRÉGOU, G. « Préfecture en béton et verre à Cergy-Pontoise ». Art. cit., p. 38.

64. - LEMOINE, Bertrand. "Les préfectures contemporaines». Monuments historiques, décembre 1991, $\mathrm{n}^{\circ} 178$, p. 35.

65. - BERNARD, Henry. "Préfecture du Val d'Oise, propos de l'architecte », tapuscrit, avril 1988 (archives de la préfecture du Val-d'Oise, [non coté]).

66. - MONNIER, Gérard. L'Architecture moderne en France, t.3, De la croissance à la compétition, 1967-1999. Paris : Picard, 2000, p. 38-39.

67. - ANDRIEUX, Jean-Yves. L'Architecture de la République. Les lieux de pouvoir dans l'espace public en France, 1792-1981. Paris : SCÉRÉN-CNDP, 2009, p. 184. 


\section{RÉSUMÉS}

Le département du Val-d'Oise fut créé en conséquence de la loi du 10 juillet 1964 qui modifiait l'administration territoriale de la région parisienne. Alors que Pontoise fut désignée comme cheflieu, les services préfectoraux s'installèrent au milieu des champs, dans la commune limitrophe de Cergy. Inaugurée en juillet 1970, la nouvelle préfecture constitua la première réalisation d'ampleur du quartier urbanisé sur dalle de Cergy-Préfecture, centre de la ville nouvelle de Cergy-Pontoise. La conception de cet édifice implanté ex nihilo fut confiée à l'architecte Henry Bernard, qui prit le parti de l'encorbellement en imaginant une vaste pyramide inversée et édifiée sur une base carrée. Agrémentée de sculptures de parement de François Stahly, d'un décor intérieur dû à Joseph-André Motte, cette préfecture constituait une tentative de renouvellement typologique. Décrite comme une « maison accueillante », où "l'accessibilité pour tous était la première volonté», elle fut un essai de dépassement de l'architecture administrative, tant par ses aménagements que par son volume et son implantation.

The Department of Val-d'Oise was created by the law of July 10, 1964, which modified the territorial administration of the Paris region. While Pontoise was designated as regional capital, the prefectural services were installed in the middle of the fields, in the neighbouring town of Cergy. The Prefecture building of Val-d'Oise was inaugurated in July 1970. This building was the first major structure within the newly urbanised neighbourhood of Cergy-Prefecture, the centre of the new town of Cergy-Pontoise. The design of this Prefecture, established ex nihilo, was commissioned from the architect Henry Bernard, who took up the challenge of a corbelled construction and imagined a huge inverted pyramid, built on a square base. Embellished by facing sculptures by François Stahly and decorated by Joseph-André Motte, this Prefecture was an attempt at a typological renewal. Described as a 'welcoming home', where 'accessibility for all was the first priority', the building was an attempt to renew administrative architecture through its interior design, volumes and localisation.

\section{INDEX}

Keywords : Cergy-Pontoise, new towns, Paris suburbs, Trente glorieuses, period of post-war prosperity, Henry Bernard, François Stahly, Joseph-André Motte, town-planning on raised platforms, Prefecture, administrative architecture, corbelled building

Mots-clés : Cergy-Pontoise, villes nouvelles, banlieue parisienne, Trente Glorieuses, Henry Bernard, François Stahly, Joseph-André Motte, urbanisme sur dalle, préfecture, architecture administrative, encorbellement 


\section{AUTEURS}

\section{LÉO NOYER-DUPLAIX}

Chercheur, service patrimoine de la Ville d'Abbeville, associé au service de l'Inventaire général du patrimoine culturel, Région Hauts-de-France ; Doctorant, Sorbonne Université, centre AndréChastel (UMR 8150) leo.nd@me.com

\section{EMMANUELLE PHILIPPE}

Conservateur du patrimoine, Région Île de France, Direction de la Culture, Service Patrimoines et Inventaire emmanuelle.philippe@iledefrance.fr 